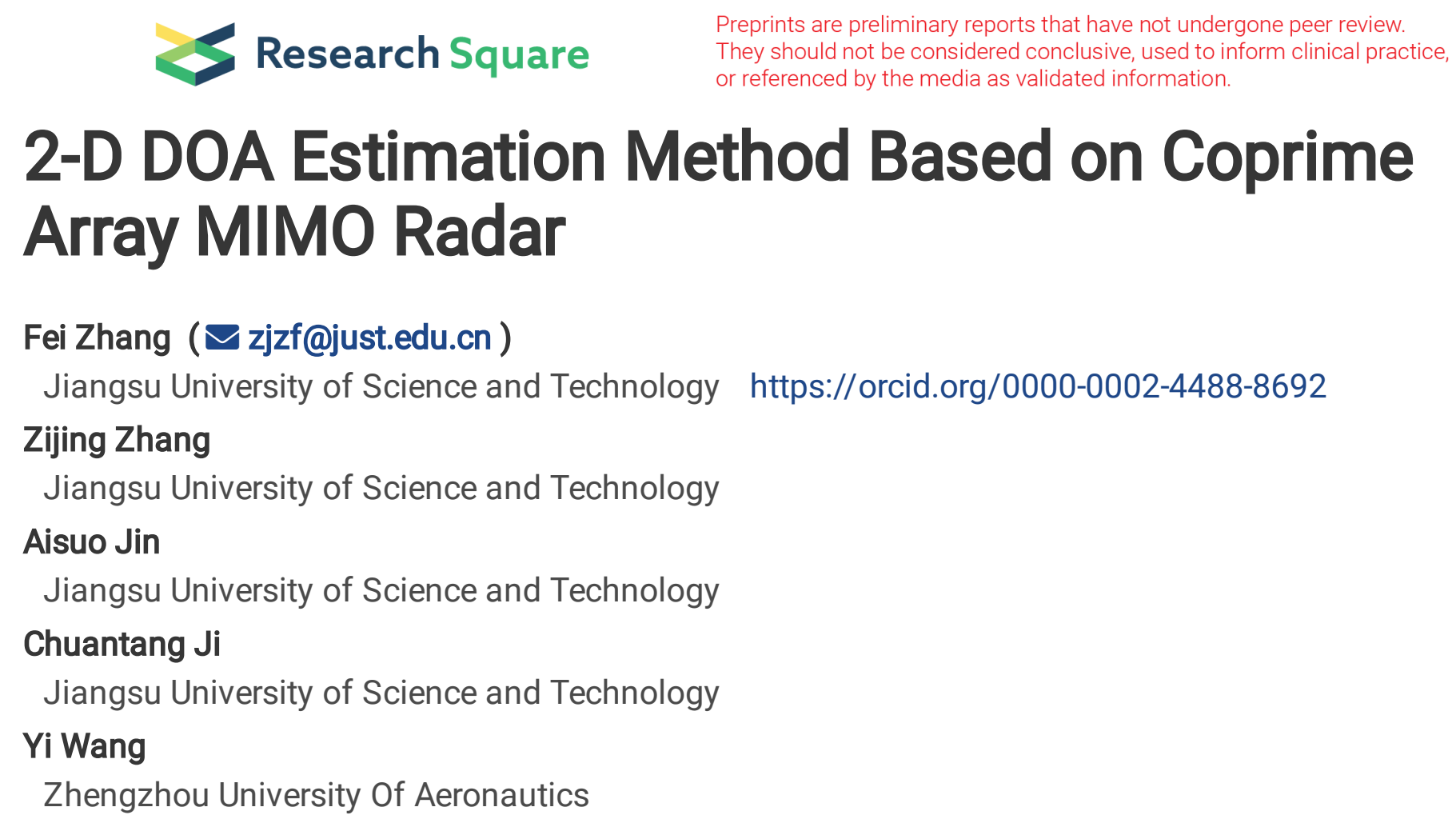

\section{2-D DOA Estimation Method Based on Coprime Array MIMO Radar}

Fei Zhang ( $\nabla$ zjzf@just.edu.cn )

Jiangsu University of Science and Technology https://orcid.org/0000-0002-4488-8692

\section{Zijing Zhang}

Jiangsu University of Science and Technology

Aisuo Jin

Jiangsu University of Science and Technology

Chuantang Ji

Jiangsu University of Science and Technology

Yi Wang

Zhengzhou University Of Aeronautics

\title{
Research
}

Keywords: Two-dimensional DOA, MIMO, coprime array, sparse array, compressed sensing

Posted Date: May 10th, 2021

DOI: https://doi.org/10.21203/rs.3.rs-478458/v1

License: (c) (i) This work is licensed under a Creative Commons Attribution 4.0 International License. Read Full License

Version of Record: A version of this preprint was published at EURASIP Journal on Advances in Signal Processing on August 18th, 2021. See the published version at https://doi.org/10.1186/s13634-02100783-x. 


\title{
2-D DOA Estimation Method Based on Coprime Array MIMO Radar
}

\author{
Fei Zhang ${ }^{1}$, Zijing Zhang ${ }^{1}$, Aisuo Jin ${ }^{1}$, Chuantang Ji ${ }^{1}$, Yi Wang ${ }^{2}$ \\ ${ }^{1}$ School of Electronic and Information, Jiangsu University of Science and Technology, \\ Zhenjiang 212003, China \\ ${ }^{2}$ School of Intelligent Engineering, Zhengzhou University of Aeronautics, Zhengzhou \\ 450046, China
}

Correspondence should be addressed to Fei Zhang; zjzf@just.edu.cn

\begin{abstract}
Aiming at the problem that traditional Direction of Arrival (DOA) estimation methods cannot handle multiple sources with high accuracy while increasing the degree of freedom, a new method of 2-D DOA estimation based on coprime array MIMO Radar (SA-MIMO-CA). Frist of all, in order to ensure the accuracy of multi-source estimation when the number of elements is finite, a new coprime array model based on MIMO (MIMO-CA) is proposed. The array model uses a special irregular array as the transmitting array and a uniform linear array as the receiving array. Besides, in order to reduce complexity and improve the accuracy of two-dimensional DOA estimation, a new twodimensional DOA estimation method based on sparse array is proposed. This method uses the sparse array topology of virtual array elements to analyze a larger number of information sources, and combines the compressed sensing method to process the sparse array, and obtains a larger array aperture with a smaller number of array elements, and improves the resolution of the azimuth angle. This method improves the DOA estimation accuracy and reduces the complexity. Finally, experiments verify the effectiveness and reliability of the SA-MIMO-CA method in improving the degree of freedom of the array, reducing the complexity, and improving the accuracy of the DOA.
\end{abstract}

Keywords: Two-dimensional DOA, MIMO, coprime array, sparse array, compressed sensing

\section{Introduction}

The traditional Direction of Arrival (DOA) method generally uses a uniform linear array, and the number of estimable target sources is less than the number of array elements. Classical methods such as MUSIC method [1,2] or ESPRIT method [3,4] use $\mathrm{N}$ array elements to estimate at most $\mathrm{N}$ 1 target signals, and the degree of freedom of the array is limited. Therefore, in the case of a certain number of array elements, how to optimize the array structure to obtain a larger array aperture to improve the DOA estimation accuracy and multi-target resolution has always been a hot issue for scholars $[2,5,6,7]$.

In recent years, with the continuous in-depth study of the array element structure, domestic and foreign scholars have proposed many non-uniform array structures $[8,9,10]$. For example, the nested array structure can estimate up to $2 \mathrm{~N}$ signal sources by using $\mathrm{N}$ array elements $[8,9,10]$. The nested array is not only easy to construct, but also easy to obtain the specific position of the array element and higher array freedom. However, because the distance between some elements in the nested array 
is very small, mutual coupling between the antennas will be caused, thereby affecting the performance of the array DOA estimation. The appearance of the co-prime array structure has solved the effect of mutual coupling between antennas in nested arrays. At the same time, the degree of freedom of the array is greatly improved by increasing the difference information of the array element position $[11,12,13,14]$. Although the traditional DOA square can easily be extended to two dimensions to deal with a planar array or a circular array, the traditional DOA method uses a parallel uniform linear array composed of several linear sub-arrays, which leads to a problem of large computational complexity. In order to improve the accuracy of two-dimensional DOA estimation, anti-interference, etc., a multi-input multiple-output (MIMO) radar is proposed. MIMO radar uses multiple antennas to transmit different waveforms and receive reflected signals from multiple targets. Therefore, it can achieve large degrees of freedom (DOF) based on waveform diversity, thereby improving spatial resolution, enhancing parameter identification, and improving target detection performance $[15,16,17,18,19]$. References $[20,21]$ combine MIMO and coprime array to estimate DOA, which improves the estimation performance of DOA, but coprime array still use uniform linear arrays to construct effective differential arrays with ideal characteristics, but this linearity Coprime array can only provide one-dimensional DOA estimation, and the accuracy of twodimensional DOA estimation is not very high. Therefore, this paper proposes a new MIMO-based MIMO array combination method (MIMO-CA). The transmitting array of the array combination is a special irregular array, and the receiving array is a uniform linear array. Improve the accuracy of two-dimensional DOA estimation.

Reference [22] proposed the use of compressed sensing for sparse matrix processing, which reduces the computational complexity of DOA estimation, but it is not used in the MIMO coprime array structure. Reference [23] proposes a fast DOA estimation method with parallel uniform linear arrays, which constructs a sub-array, but when there are many sources, additional matching is required, and the sensors are not fully utilized. References [24,25] proposed a new array, that is, each of the three parallel sub-arrays is consistent, but it is impossible to detect more sources under the same number of array elements. Therefore, on the basis of the new array combination method (MIMO-CA) proposed in this paper, this paper combines the methods of compressed sensing $[26,27,28,29]$, and proposes a new two-dimensional DOA method based on sparse matrix. First of all, this method can be realized by constructing an equivalent array of sparse array, that is, using the sparse array topology of virtual array elements to analyze a larger number of two-dimensional DOA sources, and can automatically match the corresponding azimuth and elevation angles. Secondly, by transforming the two-dimensional DOA estimation into two independent one-dimensional DOA estimation problems, only one variable can be estimated, thus reducing the computational complexity. Then, when the number of information sources is greater than or equal to the number of array elements, a virtual differential array is established, and sparse reconstruction and least squares operations are performed. The sparse matrix is processed through the compressed sensing method, so that $M+4$ array elements can identify $2 M^{2}$ Source. Finally, experiments verify the validity and reliability of SA-MIMO-CA for 2D DOA estimation.

The content of this paper is mainly structured as follows. The second section gives the array configuration and signal model of the SA-MIMO-CA method, the third section gives the DOA estimation method based on the sparse array, and the fourth section gives the SA-MIMO-CA The experimental results and analysis. 


\section{Preliminaries}

In this section, the array model and signal model of this article are mainly given.

\subsection{Array model}

As shown in Figure 1, MIMO targets 4 transmit arrays and $\mathrm{M}$ receive arrays, that is, the total number of arrays is $M+4$. Due to the nature of the MIMO array model, the array can be virtualized so that the number of virtualized arrays is $4 M$. The array arrangement of the transmitting array is shown in Fig. 1, and the receiving array is $M$ uniform linear arrays with a spacing of $2 M d$ along the $\mathrm{x}$-axis direction. Where, $d=\lambda / 2$ and $\lambda$ are wavelengths.
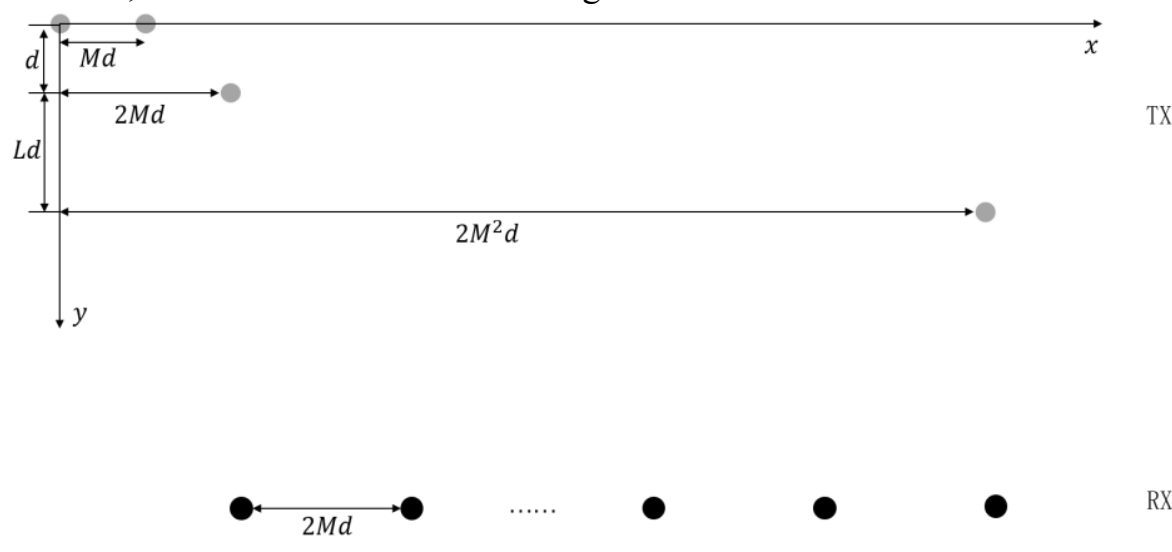

Figure 1. MIMO coprime array model

Due to the nature of the MIMO radar, the virtual array is shown in Figure 2:

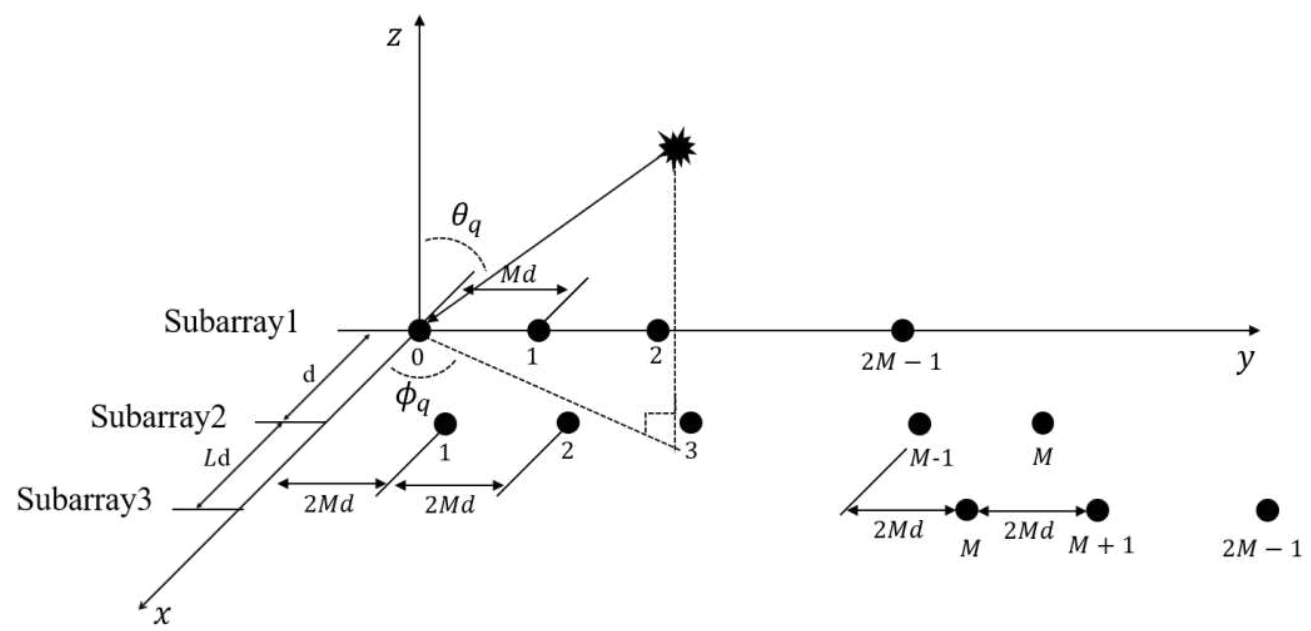

Figure 2. MIMO array model after virtual

Due to the need to construct a coprime array, the last element of sub-array 2 is discarded to form a coprime array, as shown in Figure 3: 


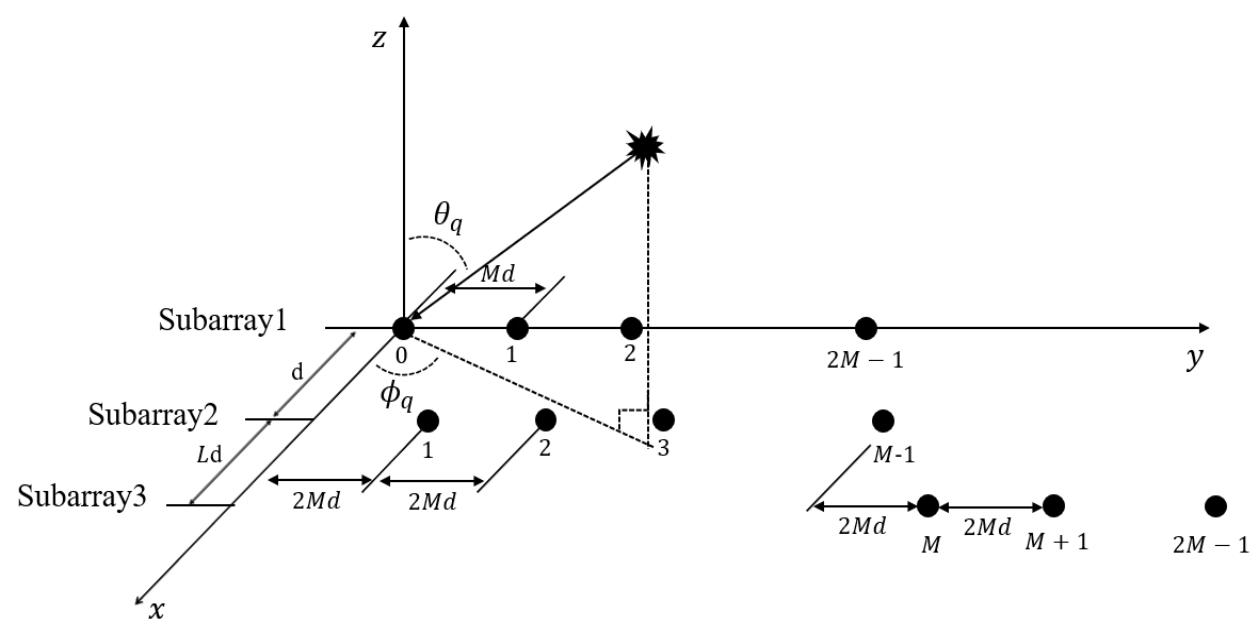

Figure 3. Virtual post-coprime MIMO array

As shown in Figure 3, the coprime array consists of three sparse linear uniform arrays. Subarray 1 has $2 M$ array elements, and its array element spacing is $M d$; and sub-arrays 2 and 3 have $M$ 1 and $\mathrm{M}$ array elements, and its array element spacing is. The array element spacing $d$ is $\lambda / 2$, where $\lambda$ is the wavelength of the corresponding carrier frequency. By choosing $M \in ¥^{+}$and $2 M \in ¥^{+}$to be relatively prime (where $¥^{+}$is expressed as a set of positive integers), the minimum cell spacing along the $y$-axis is $\lambda / 2$. This article assumes that the array sensor is located at:

$$
\left\{(x, y) \mid(0, M 2 m d) \cup\left(\mathrm{d}, 2 \mathrm{Mm}_{1} \mathrm{~d}\right) \cup\left(d+L d, 2 M^{2} d+2 M m_{2} d\right)\right\}
$$

where, $2 m \in[0,2 M-1], m_{1} \in[1, M-1], m_{2} \in[0, M-1], n, m_{1}, m_{2} \in ¥^{+}$, where (x,y) represents the coordinates in the $x-y$ plane. To make the distinction simple, let $2 M=N$. Then the array sensor is located at:

$$
\left\{(x, y) \mid(0, M 2 m d) \cup\left(\mathrm{d}, \mathrm{Nm}_{1} \mathrm{~d}\right) \cup\left(d+L d, M N d+N m_{2} d\right)\right\}
$$

\subsection{Signal model}

For the estimation of the one-dimensional wave arrival angle direction, compared with the traditional coprime array, the main difference is that these sub-arrays are no longer collinear, and are placed in parallel at distances $d$ and $L d,\left(L \in \#^{+}\right)$, that is, the minimum unit spacing $d$ along the $\mathrm{x}$-axis. As $L$ increases, the aperture of the array also increases, and the resolution also increases. But the larger the aperture, the signal will be correlated, so the value of $L$ should not be very large.

The output is:

$$
\begin{gathered}
x(l)=\left[a_{t}\left(\theta_{1}, \varphi_{1}\right) \otimes a_{r}\left(\theta_{1}, \varphi_{1}\right), a_{t}\left(\theta_{2}, \varphi_{2}\right) \otimes a_{r}\left(\theta_{2}, \varphi_{2}\right), \ldots\right. \\
\left.a_{t}\left(\theta_{k}, \varphi_{k}\right) \otimes a_{r}\left(\theta_{k}, \varphi_{k}\right)\right] S(l)+n(l)
\end{gathered}
$$

Where, $\theta_{k}$ and $\varphi_{k}$ in equation (3) are the azimuth and elevation angles of the $k$-th source, respectively. $\otimes$ is expressed as the Kronecker product. $n(l)$ is another noise vector whose elements are independently and evenly distributed in $(i, i, d)$ and obey the Gaussian distribution $C N\left(0, \sigma_{n}^{2} I_{N_{t}^{i}}\right)$, where, $i=1,2,3 . a_{t}\left(\theta_{k}, \varphi_{k}\right)=a_{t y}\left(\theta_{k}, \varphi_{k}\right) \otimes a_{t x}\left(\theta_{k}, \varphi_{k}\right), a_{r}\left(\theta_{k}, \varphi_{k}\right)=a_{r y}\left(\theta_{k}, \varphi_{k}\right) \otimes a_{r x}\left(\theta_{k}, \varphi_{k}\right)$, where $a_{t y}\left(\theta_{k}, \varphi_{k}\right)$ and $a_{t x}\left(\theta_{k}, \varphi_{k}\right)$ are the steering vectors of the transmitting array. At the same time, 
$a_{t}\left(\theta_{k}, \varphi_{k}\right) \otimes a_{r}\left(\theta_{k}, \varphi_{k}\right)$ corresponds to the Kronecker product of the receiving direction vector and the sending direction vector of the $k$-th target. $a_{t y}\left(\theta_{k}, \varphi_{k}\right) \otimes a_{t x}\left(\theta_{k}, \varphi_{k}\right)$ and $a_{r y}\left(\theta_{k}, \varphi_{k}\right) \otimes a_{r x}\left(\theta_{k}, \varphi_{k}\right)$ are the same. Let $a_{t i}\left(\theta_{q}, \varphi_{q}\right) \otimes a_{r i}\left(\theta_{q}, \varphi_{q}\right)=a_{i}\left(\theta_{q}, \phi_{q}\right)$, suppose the relationship after the virtual is:

$$
x_{i}(t)=\sum_{q=1}^{\mathrm{Q}} a\left(\theta_{q}, \phi_{q}\right) e^{j 2 \pi \frac{x_{i}}{\lambda} \sin \left(\theta_{q}\right) \cos \left(\phi_{q}\right)} s_{q}(t)+n_{i}(t)
$$

Where,

$$
a_{i}\left(\theta_{q}, \phi_{q}\right)=\left[e^{j 2 \pi \frac{y_{1}^{i}}{\lambda} \sin \left(\theta_{q}\right) \cos \left(\phi_{q}\right)}, \ldots, e^{j 2 \pi \frac{y_{t_{t}^{i}}^{i} \sin \left(\theta_{q}\right) \cos \left(\phi_{q}\right)}{\lambda}}\right]^{T}
$$

Equation (5) represents $\left(\theta_{q}, \phi_{q}\right)$ corresponding to the steering vector of the $i$-th sub-array, where $q=1, \ldots, Q, i=1,2,3 . y_{j}^{i}, 1 \leq j \leq N_{t}^{i}$ is the y coordinate of the $i$-th sensor. Where $N_{t}^{i}$ is the total number of sensors in the $i$-th sub-array, that is, $N_{t}^{1}=2 M, N_{t}^{2}=M-1, N_{t}^{3}=M$.Similarly, $x_{i}$ represents the position of the $i$-th sub-array along the x-axis, and the noise vector element is in the $i$-th sub-array $n_{i}(t)$, where $(i, i, d)$ is independently and uniformly distributed and obeys the Gaussian distribution $C N\left(0, \sigma_{n}^{2} I_{N_{t}^{i}}\right)$, where $i=1,2,3$ is. In order to transform the two-dimensional DOA estimation problem into two independent one-dimensional problems, as shown in Figure 4, $\alpha_{q}, \beta_{q} \in\left[0^{\circ}, 180^{\circ}\right]$, where $q=1, \ldots, Q$, respectively, are expressed as the angle between the incident direction and the $\mathrm{y}$-axis and $\mathrm{x}$-axis The relationship between $\alpha_{q}, \beta_{q}$ and $\theta_{q}, \phi_{q}$ is:

$$
\begin{aligned}
& \cos \left(\alpha_{\mathrm{q}}\right)=\sin \left(\theta_{q}\right) \sin \left(\phi_{q}\right) \\
& \cos \left(\beta_{\mathrm{q}}\right)=\sin \left(\theta_{q}\right) \cos \left(\phi_{q}\right)
\end{aligned}
$$

Therefore, the data vector received in the equation (4) is:

$$
x_{i}(t)=\sum_{q=1}^{Q} a_{i}\left(\alpha_{q}\right) e^{j 2 \pi \frac{x_{i}}{\lambda} \cos \left(\beta_{q}\right)} s_{q}(t)+n_{i}(t)
$$

The corresponding steering vector is:

$$
a_{i}\left(\alpha_{q}\right)=\left[e^{j 2 \pi \frac{y_{i}}{\lambda} \cos \left(\alpha_{q}\right)}, \ldots, e^{j 2 \pi \frac{y_{N_{t}}^{i}}{\lambda} \cos \left(\alpha_{q}\right)}\right]^{T}
$$

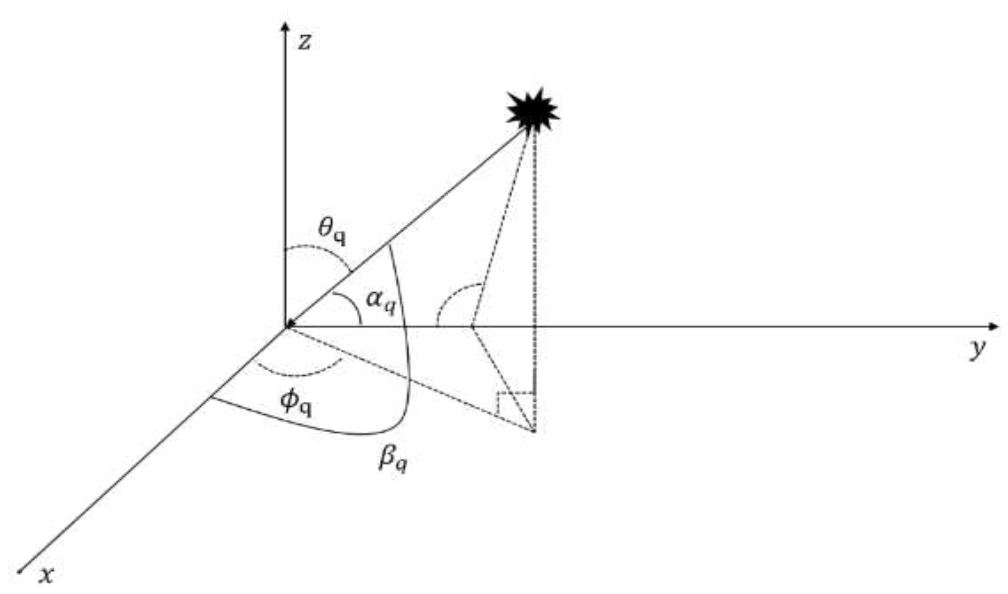

Figure 4. $\left(\theta_{q}, \phi_{q}\right)$ and $\left(\alpha_{q}, \beta_{q}\right)$ relationship diagram 
Set $s(t)=\left[s_{1}(t), \ldots, s_{Q}(t)\right]^{T}$ to the signal vector, $A_{i}=\left[a_{i}\left(\alpha_{1}\right), \ldots, a_{i}\left(\alpha_{Q}\right)\right]$ is the array manifold corresponding to the $i$-th sub-array, where $i=1,2,3$, the data vector of the receiving channel can be written as:

$$
x_{i}(t)=A_{i} B_{i} s(t)+n_{i}(t)
$$

The diagonal matrix is expressed as:

$$
B_{i}=\operatorname{diag}\left(\left[e^{j 2 \pi \frac{x_{i}}{\lambda} \cos \left(\beta_{1}\right)}, \ldots, e^{j 2 \pi \frac{x_{i}}{\lambda} \cos \left(\beta_{Q}\right)}\right]\right)
$$

Although traditional methods can achieve high-resolution DOA estimation, the $Q<N_{t} Q$ conditions must be met to obtain the noise subspace. In application, the problem of detecting information sources with more than the number of array elements has become the focus of research. In this section, an effective method is proposed to achieve the equivalence of differential arrays with a larger number of degrees of freedom. In addition, the group sparse array technology is used to improve the estimation accuracy of DOA, and the differential covariance equations of $x_{i}(t)$ and $x_{k}(t)$ are constructed.

The cross-covariance matrix of the data vectors accepted by subarrays $x_{i}(t)$ and $x_{k}(t), 1 \leq i, k \leq 3$ can be obtained:

$$
\begin{aligned}
R_{x_{i k}}= & E\left[x_{i}(t) X_{k}^{H}(t)\right] \\
& =\sum_{q=1}^{Q} \sigma_{q}^{2} e^{j 2 \pi \frac{\left(x_{i}-x_{k}\right)}{\lambda}} a_{i}\left(\alpha_{q}\right) a_{k}^{H}\left(\alpha_{q}\right)+n_{i}(t) n_{k}^{H}(t) \\
& =\left\{\begin{array}{cc}
A_{i} R_{s s} D_{i k} A_{k}^{H} & i \neq k \\
A_{i} R_{s s} A_{i}^{H}+\sigma_{n}^{2} I_{N_{t}^{i}}, i=k
\end{array}\right.
\end{aligned}
$$

Where, $R_{s}=E\left[s(t) s^{H}(t)\right]=\operatorname{diag}\left(\left[\sigma_{1}^{2}, \ldots, \sigma_{Q}^{2}\right]\right)$ is the covariance matrix of the $Q \times Q$ dimensional signal, and its diagonal term represents the scattered power of the signal. In addition:

$$
D_{i k}=B_{i} B_{k}^{H}=\operatorname{diag}\left\{\left[e^{j 2 \pi \frac{\left(x_{i}-x_{k}\right)}{\lambda} \cos \left(\beta_{1}\right)}, \ldots, e^{j 2 \pi \frac{\left(x_{i}-x_{k}\right)}{\lambda} \cos \left(\beta_{Q}\right)}\right]^{T}\right\}
$$

When $i=k$, it becomes the identity matrix.

The matrix $R_{x_{i k}}$ is quantized to obtain the following measurement vector:

$$
z_{i k}=\operatorname{vec}\left(R_{x_{i k}}\right)=\left\{\begin{array}{c}
\bar{A}_{i k} b_{i k}, \quad i \neq k \\
\bar{A}_{i k} b_{i k}+\sigma_{n}^{2} i, i=k
\end{array}\right.
$$

Where,

$$
\begin{gathered}
\bar{A}_{i k}=\left[\bar{a}_{i k}\left(\alpha_{1}\right), \ldots, \bar{a}_{i k}\left(\alpha_{Q}\right)\right] \\
b_{i k}=\left[\sigma_{1}^{2} e^{j 2 \pi \frac{\left(x_{i}-x_{k}\right)}{\lambda} \cos \left(\beta_{1}\right)}, \ldots, \sigma_{1}^{2} e^{j 2 \pi \frac{\left(x_{i}-x_{k}\right)}{\lambda} \cos \left(\beta_{Q}\right)}\right]^{T}
\end{gathered}
$$

Where, $\bar{a}_{i k}\left(\alpha_{q}\right)=a_{i}\left(\alpha_{q}\right) \otimes a_{k}^{*}\left(\alpha_{q}\right), 1 \leq q \leq Q, \quad(\mathrm{~g})^{*}$ are denoted as conjugate. $i=\operatorname{vec}\left(I_{N_{t}^{i}}\right)$, using the van der Monte structure of vectors $a_{i}\left(\alpha_{q}\right)$ and $a_{k}\left(\alpha_{q}\right)$, the entry in $\bar{a}_{i k}\left(\alpha_{q}\right)$ retains the $e^{j \pi(M n-N m)} \cos \left(\alpha_{q}\right)$ factor. Therefore, $z_{i k}$ can be regarded as a data vector received from a single 
snapshot signal vector $b_{i k}$, and the array manifold $A_{i k}$ corresponds to a virtual array whose virtual elements are located in the self-hysteresis and cross-lag between different sub-array sets. Due to the relative prime properties of $M$ and $N$, there are fewer redundant elements in these virtual arrays. Therefore, the degree of freedom in the common array is greatly increased, so that more sources of $N_{t}$ can be estimated with fewer array elements.

\section{Methods}

On the basis of the array model and signal model proposed in the second section, this section proposes a two-dimensional DOA estimation method based on a sparse array to ensure the performance of fine processing of multiple sources while increasing the degree of freedom.

\subsection{2-D DOA estimation method for sparse array}

Based on the MIMO-CA array model and signal model mentioned in Chapter 2, the signal vector in equation (14), $Z_{i k}, 1 \leq i, k \leq 3$, can be sparsely expressed on the entire discrete angle grid as:

$$
z_{i k}=\left\{\begin{array}{c}
\bar{A}_{i k}^{\mathrm{o}} b_{i k}^{\mathrm{o}}, \quad i \neq k \\
\bar{A}_{i k}^{\mathrm{o}} b_{\mathrm{i} k}^{\mathrm{o}}+\sigma_{n}^{2} i, i=k
\end{array}\right.
$$

Where $\bar{A}_{i k}^{\mathrm{o}}$ is defined as the grid $\alpha_{g}, g=1, \ldots, G_{\alpha}$ where $\bar{a}_{i k}\left(\alpha_{g}\right)$ is located, where $G_{\alpha} ? Q$; $b_{i k}^{\mathrm{o}}$ is a sparse vector, and its non-zero entry position corresponds to the DOA estimated by $\alpha_{q}$, where, $q=1, \ldots, Q$. For different sub-arrays, non-zero items usually have different values, but share the same position when searching. In other words, $b_{i k}^{o}$ exhibits a set of sparsity on all pairs of subarrays. Therefore, the estimation of $\alpha_{q}, q=1, \ldots, Q$ can be solved in the sparse reconstruction framework [22], making full use of all the degrees of freedom of mutual lag and cross lag. Many effective methods in the framework of convex optimization [26-27] and Bayesian sparse learning [28] can be used to solve the sparse reconstruction problem of complex-valued groups [28-29]. In this paper, the complex multi-task Bayesian compressed sensing method is introduced into the SAMIMO-CA method, mainly because the method has superior performance and robustness to solve the coherence problem, as follows:

In order to use self-lag and cross-lag, this paper re-planned the vector $Z_{i k}$ :

$$
z_{i k}=\Phi_{i k}^{\mathrm{o}} b_{i k}^{\mathrm{o}}+\varepsilon_{i k}, 1 \leq i, k \leq 3
$$

The respective steering matrix of each vector is:

$$
\Phi_{i k}^{\mathrm{o}}=\left\{\begin{array}{l}
{\left[\bar{A}_{i k}^{\mathrm{o}}, i\right], \quad i=k} \\
{\left[\bar{A}_{i k}^{\mathrm{o}}, 0_{N_{t}^{i} N_{t}^{k} \times 1}\right], i \neq k}
\end{array}\right.
$$

The dimensionality of the unknown sparse vector is extended to $\bar{b}_{i k}^{\mathrm{o}}$, and an additional element with 
a noise power of $\sigma_{n}^{2}$ is required. In this case, use $\bar{b}_{i k}^{\mathrm{o}}$ to estimate that the first $G_{\alpha}$ is used to determine $\alpha_{q}$, and discard the last element. In addition, the error vector $\varepsilon_{i k}$ is included in (18) to illustrate the difference between the statistical expectation and the sample average when calculating the covariance matrix. The difference is modeled as $i, i, d$, and since a sufficient number of samples are used in the averaging, a Gaussian complex number is produced.

Suppose the elements in $\bar{b}_{i k}^{\circ}$ come from the product of the following zero-mean Gaussian distribution:

$$
\bar{b}_{i k}^{\mathrm{og}}: N\left(\bar{b}_{i k}^{\mathrm{og}} \mid 0, \gamma_{g} I_{2}\right), g \in\left[1, \ldots, G_{\alpha}\right]
$$

Where, $N(x \mid a, b)$ means that the random variable $\mathrm{x}$ follows the Gaussian distribution and the

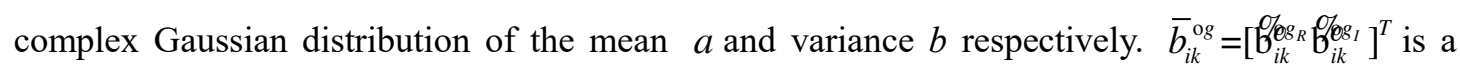
$2 \times 1$ vector composed of the real part coefficient $b_{i k}^{b g_{R}}$ and the imaginary part coefficient $b_{i k}^{b g_{I}}$, corresponding to the $g$-th grid. It can be easily determined that when $\gamma_{g}$ is set to $0, \bar{b}_{i k}^{\text {og }}$ approaches zero $[30,31,32]$. To achieve the sparsity of $\bar{b}_{i k}^{\mathrm{o}}$, a Gamma prior is set to $\gamma_{g}^{-1} \sim \operatorname{Gamma}\left(\gamma_{g}^{-1} \mid a, b\right)$, where $\operatorname{Gamma}\left(x^{-1} \mid a, b\right)=\Gamma(a)^{-1} b^{a} x^{-(a-1)} e^{-\frac{b}{x}}$ and $\Gamma(\mathrm{g})$ are Gamma functions. $a$ and $b$ are hyperparameters. The vector $\gamma=\left[\gamma_{1}, \ldots, \gamma_{G}\right]^{T}$ contains $\bar{b}_{i k}^{\text {og }}$, where $g=1, \ldots, G_{\alpha}$ is shared with all groups to enhance sparsity. Similarly, the Gaussian prior is $N\left(0, \xi_{0} I_{2}\right), \varepsilon_{i k}$ is set, and the Gamma prior is on $\xi_{0}^{-1}$, with hyperparameters $c$ and $d$. Defining the density function of the two $G_{a} \times 1$ vectors $\bar{b}_{i k}^{\mathrm{o} R}=\left[\mathrm{b}_{i k}^{\mathrm{olk}}, \ldots, \mathrm{b}_{i k}^{\mathrm{oGR}}\right]^{T}$ and $\bar{b}_{i k}^{\mathrm{o}_{l}}=\left[\mathrm{b}_{i k}^{\mathrm{ol} I}, \ldots, \mathrm{b}_{i k}^{\mathrm{oGI}}\right]^{T}$ associated as $\bar{b}_{i k}^{\mathrm{o}_{\mathrm{ol}}}=\left[\left(\bar{b}_{i k}^{\mathrm{o}_{\mathrm{R}}}\right)^{T},\left(\bar{b}_{i k}^{\mathrm{o}_{\mathrm{o}}}\right)^{T}\right]^{T}$ can be evaluated as:

$$
P_{r}\left(\bar{b}_{i k}^{\mathrm{o}_{R I}} \mid \bar{z}_{i k}, \Phi_{i k}^{\mathrm{o}}, \gamma, \xi_{0}\right)=\mathcal{N}\left(\bar{b}_{i k}^{\mathrm{o}_{R I}} \mid \mu_{i k}, \sum_{i k}\right)
$$

Where,

$$
\begin{aligned}
& \bar{z}_{i k}^{R I}=\left[\operatorname{Re}\left(z_{i k}\right)^{T}, \operatorname{Im}\left(z_{i k}\right)^{T}\right]^{T} \\
& \mu_{i k}=\xi_{0}^{-1} \Sigma_{i k} \Psi_{i k}^{T} \bar{z}_{i k}^{R I} \\
& \Sigma_{i k}=\left[\xi_{0}^{-1} \Psi_{i k}^{T} \Psi_{i k}+F^{-1}\right]^{-1} \\
& \Psi=\left[\begin{array}{cc}
\operatorname{Re}\left(\Phi_{i k}^{\mathrm{o}}\right) & -\operatorname{Im}\left(\Phi_{i k}^{\mathrm{o}}\right) \\
\operatorname{Im}\left(\Phi_{i k}^{\mathrm{o}}\right) & \operatorname{Re}\left(\Phi_{i k}^{\mathrm{o}}\right)
\end{array}\right] \\
& F=\operatorname{diag}\left(\gamma_{1}, \ldots, \gamma_{G}, \gamma_{1}, \ldots, \gamma_{G_{\alpha}}\right)
\end{aligned}
$$

Obviously, when $\gamma$ and $\xi_{0}$ are given, (23) and (24) can be used to derive the mean and variance of each scattering system in $\bar{b}_{i k}^{\text {oRI }}$. On the other hand, the values of $\gamma$ and $\xi_{0}$ are determined by maximizing the logarithm of the edge likelihood, which can be achieved by the expectation maximization method to produce: 


$$
\begin{gathered}
\gamma_{g}^{(\text {new })}=\frac{1}{9} \sum_{i, k=1}^{3}\left(\operatorname{Tr}\left[\sum_{i k} \Psi_{i k}^{T} \Psi_{i k}\right]+\left\|g_{i k}^{R I}-\Psi_{i k} \mu_{i k}\right\|_{2}^{2}\right) \\
\xi_{0}^{(\text {new })}=\frac{1}{18 G_{\alpha}} \sum_{i, k=1}^{3}\left(\operatorname{Tr}\left[\sum_{i k} \Psi_{i k}^{T} \Psi_{i k}\right]+\left\|q_{i k}^{R I}-\Psi_{i k} \mu_{i k}\right\|_{2}^{2}\right)
\end{gathered}
$$

Where $\mu_{i k, g}$ and $\mu_{i k, g+G_{\alpha}}$ are the $g$-th and $g+G_{\alpha}$-th elements of the $\mu_{i k}$ vector, and $\Sigma_{i k, g g}$ and $\left.\sum_{i k,\left(g+G_{\alpha}\right)\left(g+G_{\alpha}\right.}\right)$ are the $(g, g)$ and $\left(g+G_{\alpha}, g+G_{\alpha}\right)$-th elements in the matrix $\Sigma_{i k}$, because $\gamma$ and $\xi_{0}$ depend on $\mu_{i k}$ and $\Sigma_{i k}$. Since CMT-BCS is iterative, iterate between (22)-(24) and (27)(28) until the convergence criterion is reached. The estimated value $\hat{\alpha}_{q}, q=1, \ldots, Q$ can obtain the maximum value of $Q$ in $\sum_{i, k=1}^{3}\left(b_{i k}^{\text {ogR }}+b_{i k}^{\text {ogI }}\right), g=1, \ldots, G$. Then the $Q \times 1$ vector in (14), that is, $b_{i k}, i \neq k$ can be estimated by least squares fitting, expressed as:

$$
\hat{b}_{i k}=\left(\hat{\bar{A}}_{i k}^{H} \hat{\bar{A}}_{i k}\right) \hat{\bar{A}}_{i k}^{H} z_{i k}, i \neq k
$$

Where,

$$
\bar{A}_{i k}=\left[a_{i k}\left(\hat{\alpha}_{1}\right), \ldots, \bar{a}_{i k}\left(\hat{\alpha}_{1}\right)\right]
$$

Therefore, $\beta_{q}, q=1, \ldots, Q$ estimates:

$$
\hat{\beta}_{q}=\cos ^{-1}\left(-\operatorname{phase}\left(\hat{b}_{q}\right) / \pi\right)
$$

Where, $\hat{b}_{q}$ is the q-th element of vector $\hat{b}_{i k}$, so $\hat{\beta}_{q}$ automatically matches $\hat{\alpha}_{q}$, and $\hat{\alpha}_{q}$ can be obtained in the same way, so by equations (6) and (7):

$$
\begin{gathered}
\hat{\theta}_{q}=\sin ^{-1}\left[\sqrt{\cos ^{2}\left(\hat{\alpha}_{q}\right)+\cos ^{2}\left(\hat{\beta}_{q}\right)}\right] \\
\hat{\phi}_{q}=\tan ^{-1}\left[\frac{\cos \left(\hat{\alpha}_{q}\right)}{\cos \left(\hat{\beta}_{q}\right)}\right]
\end{gathered}
$$

\subsection{Steps of the SA-MIMO-CA method}

The steps of the SA-MIMO-CA method are as follows:

Step1: Construct the MIMO array model to obtain the MIMO coprime array model in Figure 1.

Step2: To obtain a coprime array, discard the last element of the second row of the virtual array, and get equation (3).

Step3: Use equations (6) and (7) to turn the two-dimensional problem into two independent onedimensional DOA estimation problems.

Step4: Use equation (12) to obtain the cross-covariance matrix $R_{x_{i k}}$ of the signal.

Step5: Use equation (14) to obtain the quantized measurement vector $z_{i k}$.

Step6: Use equation (18) to obtain the re-planned $z_{i k}$.

Step7: Use equations (29) and (30) to obtain $\hat{b}_{i k}$.

Step8: The two-dimensional DOA estimation of the $N_{t}$ signals are obtained by matching equations (32) and (33). 


\section{Results and discussion}

This section mainly gives the experimental results and analysis. In this section, first of all, it is verified through experiments that the method in this paper is effective and reliable in improving the degree of freedom. Besides, it is verified through experiments that this article is effective and reliable in improving the performance of 2-D DOA estimation.

\subsection{Degree of freedom analysis}

In the case of one-dimensional, the obtained co-array is equivalent to the traditional coprime array, that is, the number of estimated signals can reach: $Q_{a v}=M N$, that is, $Q_{a v}=M^{2}$. For a given number of physical antennas $N_{t}=2 M+N-1=4 M-1, Q_{a v}$ can be obtained in the following way:

The maximum number of sources can be estimated: $Q_{a v}=M N=M^{2}$

$$
\begin{aligned}
& \text { Restricted to } N_{t}=2 M+N-1=4 M-1 \\
& M<N, M, N \in ¥^{+}
\end{aligned}
$$

Obviously, the effective optimal coprime pair is that $2 M$ and $\mathrm{N}$ are as equal as possible, that is, the array in this paper is selected as the optimal number of array elements. In this case, the maximum number of estimated signals $Q_{a v}$ is:

$$
Q_{\max }=\left[\frac{N_{t}\left(N_{t}+2\right)}{8}\right]
$$

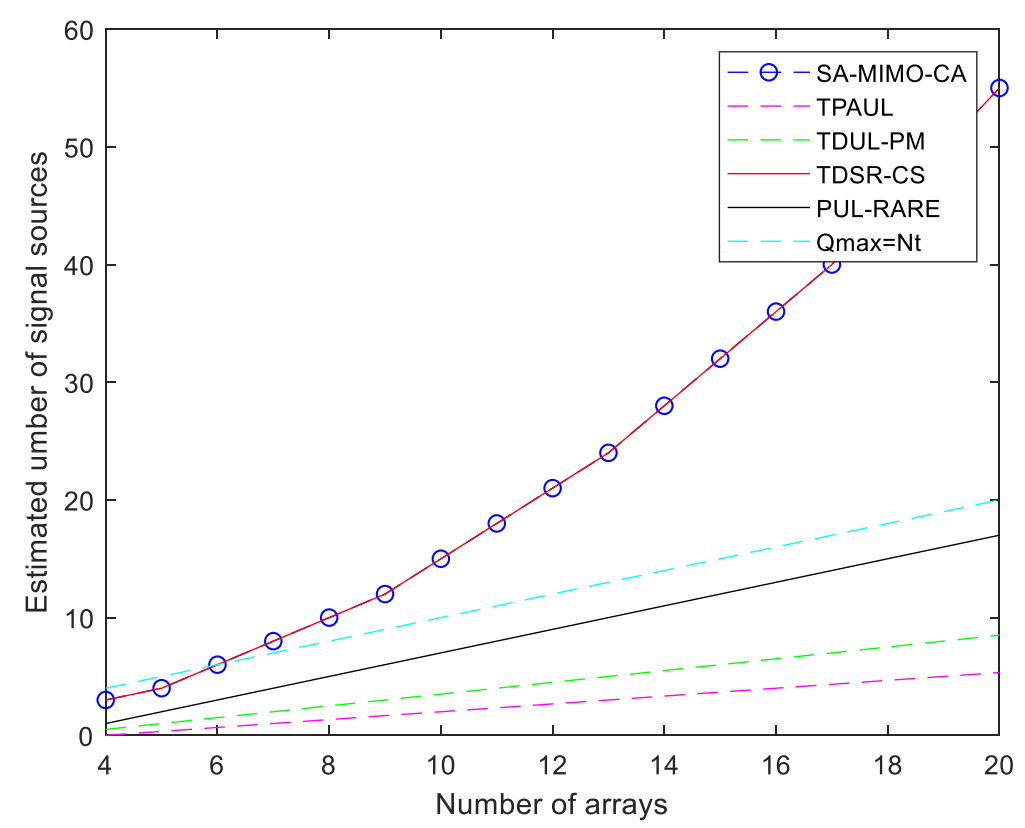

Figure 5. The relationship between the number of arrays and the number of estimable sources

As shown in Figure 5 above, we compare the $Q_{\max }$ value of the method in this paper with the TDUL-PM method, TPAUL method, PUL-RARE method, TDSR-CS in references [25,30,34,35]. The methods are compared in the figure. Although the value of $Q_{\max }$ of all methods increases with the increase of $N_{t}$, it is obvious that the method based on the co-prime array (the MIMO array 
method, TDSR-CS method, TDUL-PM method and other methods proposed in this paper) is significantly better than other methods method. When $N_{t}>6$, the method based on the relative prime array can resolve more sources than the number of other array sensors. For other methods, the number of resolvable sources is less than the number of sensors.

\subsection{2-D DOA estimation performance comparison}

\subsubsection{The relationship between signal-to-noise ratio and mean square error}

Compare the SA-MIMO-CA proposed in this article with the TPAUL method, TDUL-PM method, TDSR-CS method and PUL-RARE method in references [25,30,34,35] to verify Two-dimensional DOA estimation performance of SA-MIMO-CA. Perform 100 Monte Carlo simulations for each method, and define the root mean square error as:

$$
\begin{aligned}
& R M S E_{\theta}=\sqrt{\frac{1}{I Q} \sum_{i=1}^{I} \sum_{q=1}^{Q}\left(\hat{\theta}_{q}(i)-\theta_{q}\right)^{2}} \\
& R M S E_{\phi}=\sqrt{\frac{1}{I Q} \sum_{i=1}^{I} \sum_{q=1}^{Q}\left(\hat{\phi}_{q}(i)-\phi_{q}\right)^{2}}
\end{aligned}
$$

Where, $I$ is the number of Monte Carlo experiments, and Q is the number of sources. We set $M=4$; that is, the array configuration of the $N_{t}=4 M-1=15$ antenna. In addition, let $L=20$. Assume that $Q$ far-field sources with the same power are on the elevation plane $\left(\theta_{q}, \phi_{q}\right)$, where $\theta_{q} \in\left[0^{\circ}, 90^{\circ}\right], \phi_{q} \in\left[-90^{\circ}, 90^{\circ}\right], q=1, \ldots, Q$. The grid interval in the angular space is set to $0.1^{\circ}$, and the parameter in the Bayesian sparse learning group is set to $a=b=c=d=0$.

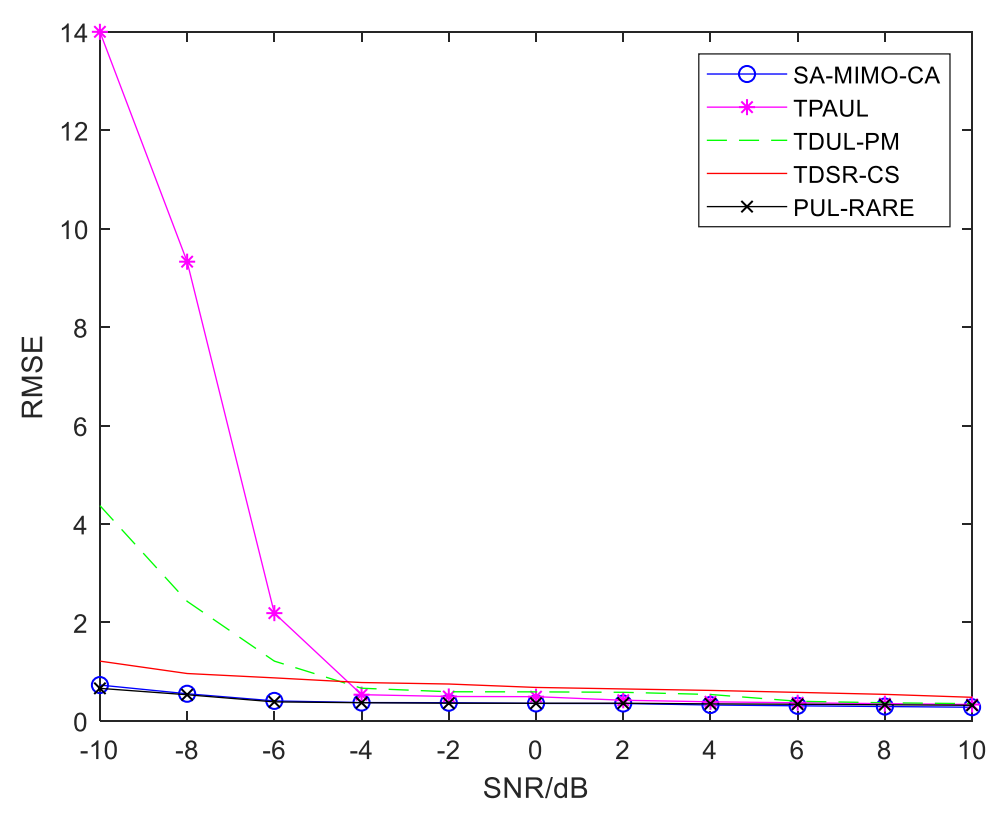

(a) The relationship between the SNR of the pitch angle and the RMSE of the pitch angle 


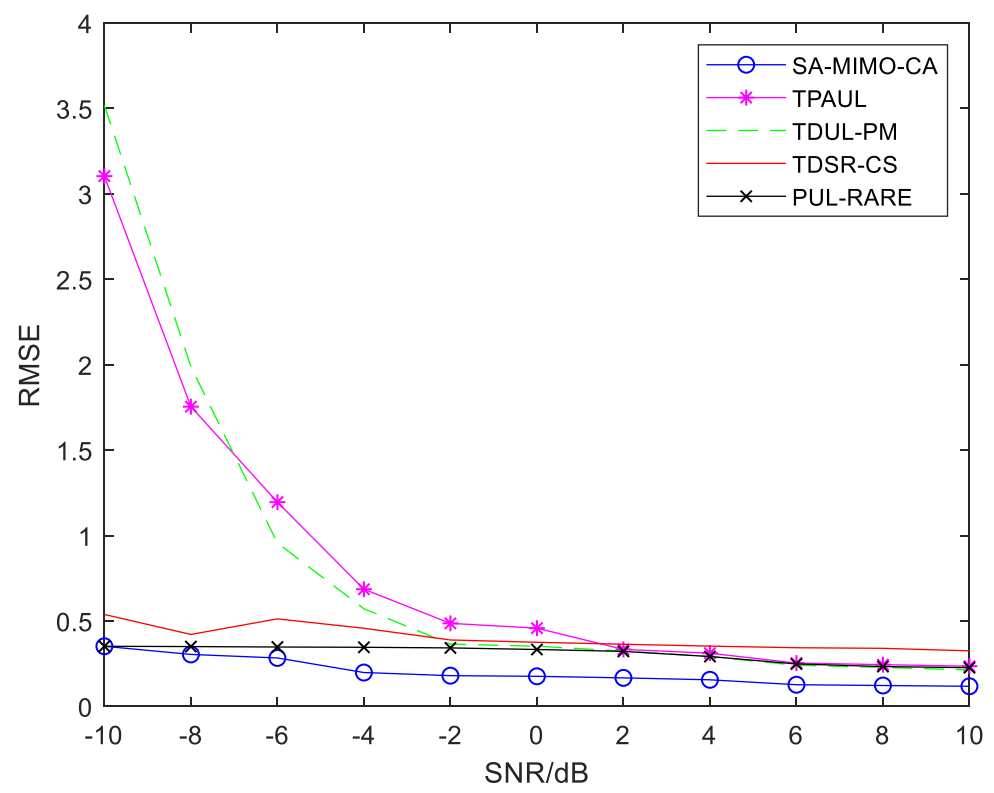

(b) The relationship between the SNR of the azimuth angle and RMSE of the azimuth angle

Figure 6. The relationship between mean square error and signal-to-noise ratio (the number of sources is 3 )

Figure 6 shows the SA-MIMO-CA method and TPAUL method, TDUL-PM method, TDSRCS method, and PUL-RARE method when the number of sources $Q=3$ and the number of snapshots $T=500$ The estimated performance is compared, and the RSME changes of the method under different signal-to-noise ratios (SNR) are investigated. At the elevation angle, the performance of SA-MIMO-CA and PUL-RARE methods are close; but at the azimuth angle, when the signal-tonoise ratio is 0 , the performance of SA-MIMO-CA is improved by about $47.1 \%$ compared with the PUL-RARE method. Compared to the TPAUL method, it has increased by about $61.5 \%$. By comparing RSME under different signal-to-noise ratios, it is concluded that SA-MIMO-CA has better estimation performance than several other methods under low signal-to-noise ratios. Specific data are shown in Table 1 and Table 2.

Table 1: The relationship between the signal-to-noise ratio of the elevation angle and the azimuth angle and the mean square error

\begin{tabular}{lccccc}
\hline & SA-MIMO-CA & TPAUL & TDSR-CS & TDUL-PM & PUL-RARE \\
\hline $\begin{array}{l}\text { Pitch angle (mean } \\
\text { value of RMSE) }\end{array}$ & 0.395 & 2.628 & 0.739 & 1.100 & 0.397 \\
$\begin{array}{l}\text { Azimuth (mean } \\
\text { value of RMSE) }\end{array}$ & 0.198 & 0.824 & 0.401 & 0.822 & 0.308 \\
\hline
\end{tabular}

Table 2: The relationship between the signal-to-noise ratio of the elevation angle and the azimuth angle and the mean square error (when the signal-to-noise ratio is 0 )

\begin{tabular}{lccccc}
\hline & SA-MIMO-CA & TPAUL & TDSR-CS & TDUL-PM & PUL-RARE \\
\hline $\begin{array}{l}\text { Mean square error of } \\
\text { pitch angle }\end{array}$ & 0.356 & 0.493 & 0.680 & 0.590 & 0.359 \\
$\begin{array}{l}\text { Azimuth mean } \\
\text { square error }\end{array}$ & 0.176 & 0.458 & 0.375 & 0.352 & 0.333 \\
\hline
\end{tabular}

\subsubsection{The relationship between angle and number of snapshots}




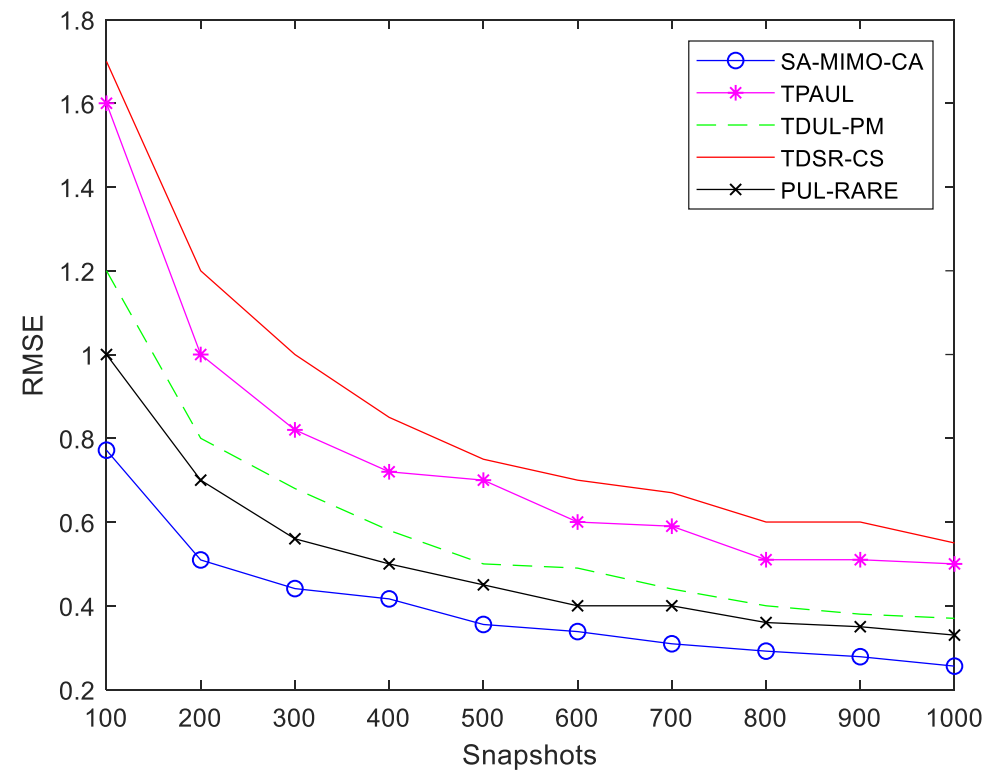

(a) The relationship between the mean square error of the pitch angle and the number of snapshots

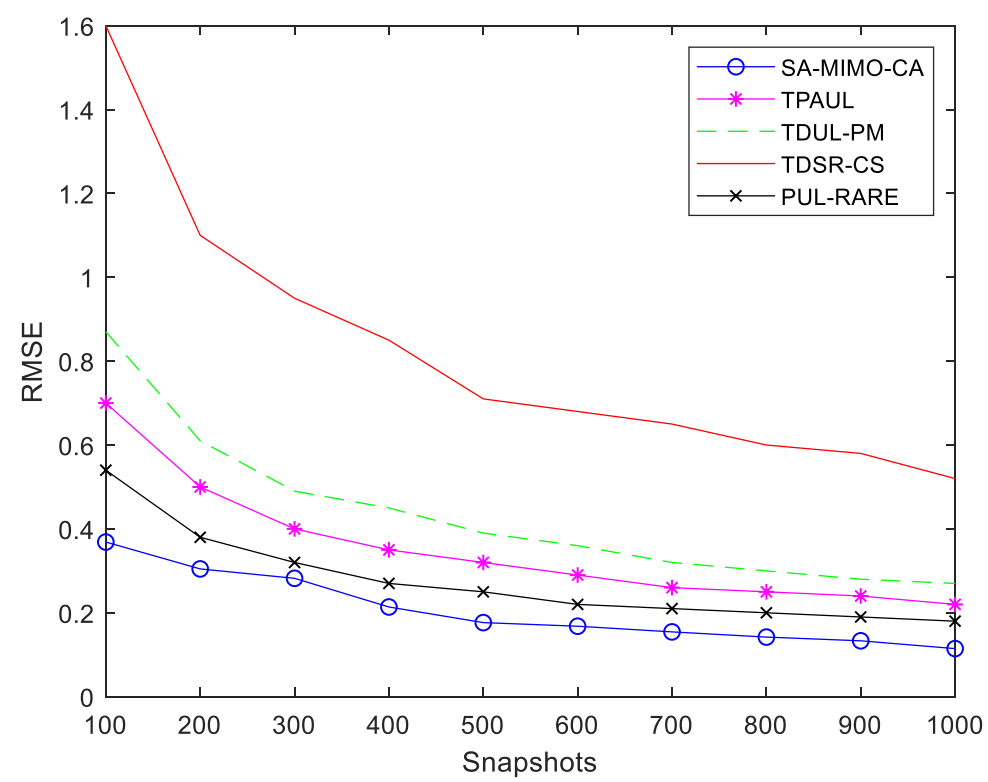

(b)The relationship between the mean square error of the azimuth angle and the number of snapshots

Figure 7. The relationship between the mean square error and the number of snapshots (the number of sources is

Figure 7 show that the SA-MIMO-CA method, TPAUL method, TDUL-PM method, TDSR-CS method, and PUL-RARE method are used to estimate the position when the number of sources $Q=3$ and $S N R=0$. The performance comparison of each method, the comparison of RSME under different snapshots. The overall performance of the SA-MIMO-CA method is improved by about $44.5 \%$ compared to the TPAUL method, and about $23.4 \%$ compared with the PUL-RARE method. Experimental results show that SA-MIMO-CA performs better than TPAUL method and PULRARE method under different snapshots.

Table 3. Relationship between pitch angle, azimuth angle and the number of snapshots 


\begin{tabular}{lccccc}
\hline & $\begin{array}{c}\text { SA-MIMO- } \\
\text { CA }\end{array}$ & TPAUL & TDSR-CS & TDUL-PM & PUL-RARE \\
\hline $\begin{array}{l}\text { Pitch angle } \\
\text { (mean value of }\end{array}$ & 0.397 & 0.755 & 0.862 & 0.584 & 0.505 \\
$\begin{array}{l}\text { RMSE) } \\
\begin{array}{l}\text { Azimuth (mean } \\
\text { value of RMSE) }\end{array}\end{array}$ & 0.206 & 0.353 & 0.824 & 0.434 & 0.276 \\
\hline
\end{tabular}

\subsubsection{Comparison of 2-D DOA estimation}

In the above two sets of experiments, the performance of the method at low signal sources was tested, and the comparison of different signal-to-noise ratios and the mean square error of different snapshots proved the superiority of the method. Next, conduct a multi-source experiment. There are $Q$ sources, the number of sources is greater than the number of arrays, the signal-to-noise ratio is kept to 0 , and the number of snapshots is set to 500 , and compared with the TDSR-CS method, as shown in Figure 8.

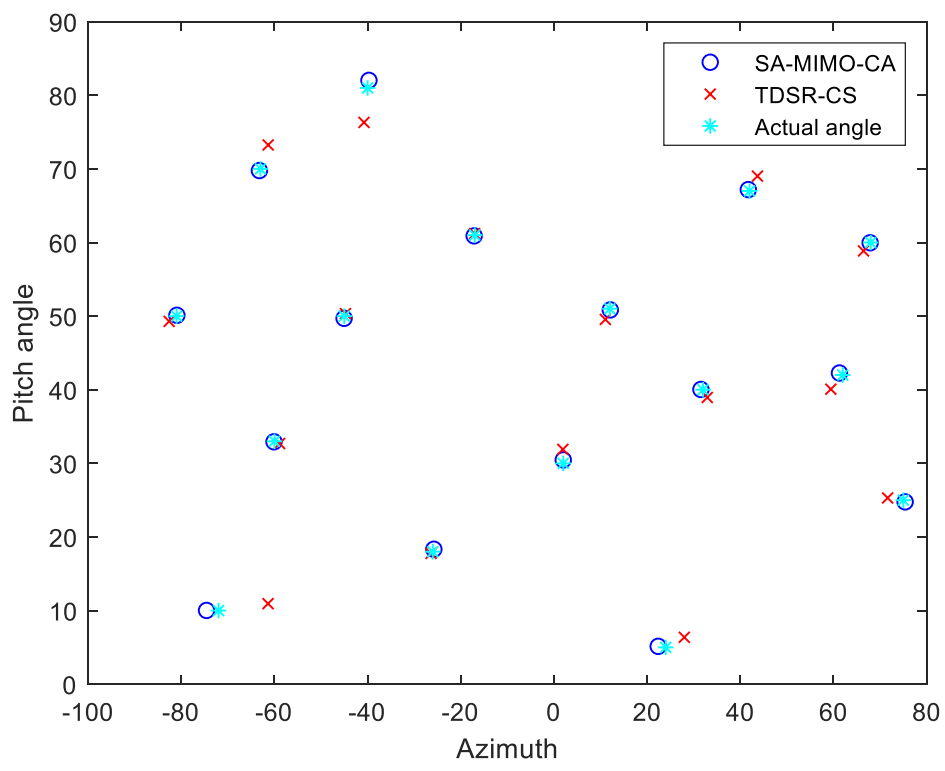

Figure 8. Comparison of two-dimensional DOA estimation

Figure 8 shows the DOA estimation performance of each method, each of which represents the SA-MIMO-CA method in this paper, the actual value and the TDSR-CS method when the signalto-noise ratio is $S N R=0$, and the number of snapshots is 500 . The DOA estimation result can be intuitively seen from the figure that SA-MIMO-CA is closer to the actual angle and has better twodimensional DOA estimation performance.

\section{Conclusion}

Aiming at the problem that traditional array signal processing methods cannot handle multiple sources with high accuracy while increasing the degree of freedom, this paper proposes a new twodimensional DOA estimation method based on MIMO radar coprime array. This method mainly uses the characteristics of coprime arrays and MIMO radars, and combines the theory of compressed 
sensing to improve the degree of freedom and accuracy of DOA estimation, and reduce the computational complexity. Through experimental verification, compared with TPAUL method, TDUL-PM method, TDSR-CS method, PUL-RARE method, this method can effectively distinguish more signal sources. And it has high two-dimensional DOA estimation accuracy, and improves the degree of freedom of two-dimensional DOA estimation.

\section{Availability of data and materials:}

The data that support the findings of this study are available from the corresponding author upon reasonable request.

\section{Funding}

This work was supported by the National Natural Science Foundation of China under Grant No.61801170 and 61801435, Jiangsu Overseas Visiting Scholar Program for University Prominent Yong \& Middle-aged Teachers and Presidents, the Scientific and Technological Key Project of Henan Province under Grant 212102210559 and in part by the Training Program for Young Scholar of Henan Province for Colleges and Universities under Grand 2020GGJS172.

\section{Authors' contributions}

ZZJ analyzed and interpreted the data regarding the SA-MIMO-CA, and was a major contributor in writing the manuscript. JAS and JCT analyzed the theory of compressed sensing and MIMO. ZF and WY provided experimental ideas. All authors read and approved the final manuscript.

\section{Acknowledgments}

The authors would like to thank Dayu Yin for his academic support, and I can finish the thesis successfully.

\section{Declarations}

\section{Competing interests}

The authors declare that they have no conflicts of interest.

\section{Ethics approval and consent to participate}

Not applicable.

\section{Consent for publication}

Not applicable

\section{Abbreviations:}

DOA: Direction of arrival

MUSIC: Multiple signal classification

ESPRIT: Estimation of signal parameters via rotational invariance technique

SNR: Signal-to-noise ratio

RSME: The root mean square error

MIMO: Multiple input multiple output

MIMO-CA: Coprime array model based on MIMO

SA-MIMO-CA: The method of 2-D DOA estimation based on coprime array MIMO Radar 


\section{References}

1. R. Schmidt. Multiple emitter location and signal parameter estimation. in IEEE Transactions on Antennas and Propagation, 34(3), 276-280.DOI: 10.1109/TAP.1986.1143830. (1986)

2. L. He, et al..Noncircular Signal DOA Estimation with Reduced Dimension MUSIC for Coprime Linear Array. 2018 4th Annual International Conference on Network and Information Systems for Computers (ICNISC) (2018).

3. R. Roy and T. Kailath. ESPRIT-estimation of signal parameters via rotational in variance techniques. in IEEE Trans. On Acoustics, Speech, and Signal Processing, ,37(7), 984-995, (1986). DOI: 10.1117/12.55606.

4. P. Ma, J. Li, F. Xu and X. Zhang. Hole-Free Coprime Array for DOA Estimation: Augmented Uniform CoArray. in IEEE Signal Processing Letters, 28, 36-40,(2021). DOI:10.1109/LSP.2020.3044019.

5. P. Pal and P. P. Vaidyanathan. Nested arrays: A novel approach to array processing with enhanced degrees of freedom. IEEE Trans. Signal Process., 58(8), 4167-4181, (2010). DOI: 10.1109/TSP.2010.2049264.

6. X. Li, J. Chen, W. Tan and W. Yang. Gridless DOA estimation method for monostatic MIMO array base on covariance matrix reconstruction. Systems Engineering and Electronics, 42(5), 969-977, (2019).

7. S. Qin, Y. D. Zhang and M. G. Amin. Improved two-dimensional DOA estimation using parallel coprime arrays. Signal processing, 172(Jul.), 107428.1-107428.9, (2020). https://doi.org/10.1016/j.sigpro.2019.107428.

8. C. Liu and P. P. Vaidyanathan. Super Nested Arrays: Linear Sparse Arrays with Reduced Mutual CouplingPart I: Fundamentals. in IEEE Transactions on Signal Processing, 64(15), pp. 3997-4012, (2016). DOI: 10.1109/TSP.2016.2558159.

9. C. Liu and P. P. Vaidyanathan. Super nested arrays: Linear sparse arrays with reduced mutual coupling-Part II: High-order extensions. in IEEE Transactions on Signal Processing, 64(16), 4203-4217, (2016). DOI: 10.1109/TSP.2016.2558159.

10. J. Liu, Y. Zhang, Y. Lu, S. Ren and S. Cao. Augmented nested arrays with enhanced DOF and reduced mutual coupling. IEEE Trans. Signal Process. 65(21), 5549-5563, (2017). DOI: 10.1109/tsp.2017.2736493.

11. G. Wang, Z. Fei and S. Ren. 2D DOA Estimation Exploiting Vertical Synthetic Planar Arrays. in IEEE Access, 9, 3497-3507.DOI: 10.1109/ACCESS.2020.3047686, (2021).

12. Z. Zhang, Y. Guo, Y. Huang and P. Zhang A 2-D DOA Estimation Method with Reduced Complexity in Unfolded Coprime L-Shaped Array. in IEEE Systems Journal,15(1), 407-410, (2021). DOI: 10.1109/JSYST.2019.2948089.

13. J. Li, D. Jiang and X. Zhang. DOA Estimation Based on Combined Unitary ESPRIT for Coprime MIMO Radar. in IEEE Communications Letters, 21(1), 96-99, (2017). DOI: 10.1109/LCOMM.2016.2618789.

14. Z. Meng, and W. Zhou. Robust adaptive beamforming for coprime array with steering vector estimation and covariance matrix reconstruction. IET Communications, 14. 2749-2758, (2020). DOI: 10.1049/ietcom.2019.1314

15. Li, G., Li, T., Xu, M. et al. Sparse massive MIMO-OFDM channel estimation based on compressed sensing over frequency offset environment. EURASIP J. Adv. Signal Process. 2019,31 (2019). https://doi.org/10.1186/s13634-019-0627-3.

16. Zhou, Y., Chen, X., Li, Y. et al. A fast STAP method using persymmetry covariance matrix estimation for clutter suppression in airborne MIMO radar. EURASIP J. Adv. Signal Process. 2019, 13 (2019). https://doi.org/10.1186/s13634-019-0610-z

17. J. Li and P. Stoica. MIMO radar with colocated antennas. IEEE Signal Process. Mag., 24 (5) 106-114, (2007). DOI: $10.1109 / \mathrm{MSP} .2007 .904812$.

18. F. Wen, Z. Zhang and X. Zhang. CRBs for direction-of-departure and direction-of-arrival estimation in collocated MIMO radar in the presence of unknown spatially coloured noise. IET Radar Sonar Navig.,13 (4), 
530-537, (2019). DOI: 10.1049/iet-rsn.2018.5386.

19. F. Wen. Computationally efficient DOA estimation algorithm for MIMO radar with imperfect waveforms. IEEE Commun. Lett., 23 (6), 1037-1040, (2019). DOI: 10.1049/iet-rsn.2018.5386

20. J. Li, L. He, Y. He and X. Zhang. Joint direction of arrival estimation and array calibration for coprime MIMO radar. Digital Signal Processing, 94, 67-74, (2019). DOI: 10.1016/j.dsp.2019.07.002

21. W. Zhou, Q. Wang and J. Wang. DOA estimation for monostatic MIMO radar based on unfolded coprime array. Journal of Nanjing University of Posts and Telecommunications: Natural Science Edition, 39(6):1-8, (2019). DOI: $10.1049 / \mathrm{el} .2016 .3818$.

22. R. Bautista and J. R. Buck. Detecting Gaussian Signals Using Coprime Sensor Arrays in Spatially Correlated Gaussian Noise. IEEE Transactions on Signal Processing, 67(5), 1296-1306, (2019). DOI: 10.1109/TSP.2018.2887399.

23. W. So. A fast algorithm for 2-D direction-of-arrival estimation. Signal Processing. (2003). DOI: 10.1016/S0165-1684(03)00118-X.

24. N. Tayem and H. M. Kwon. Azimuth and elevation angle estimation with no failure and no eigen decomposition. Signal Processing, 86(1),8-16, (2006).

25. H. Chen, C. Hou, Q. Wang, et al. Improved Azimuth/Elevation Angle Estimation Algorithm for Three-Parallel Uniform Linear Arrays. IEEE Antennas \& Wireless Propagation Letters, 14,329-332, (2015). DOI: 10.1109/LAWP.2014.2360419.

26. D. L. Donoho. Compressed sensing. IEEE Trans. Inf. Theory, 52(4), 1289-1306, (2006).DOI: 10.1109/TIT.2006.871582

27. M. Yuan. Model Selection and Estimation in Regression with Grouped Variables. J. r. stat. soc, 68(1),49-67, (2006).

28. S. Ji, D. Dunson and L. Carin. Multitask compressive sensing. IEEE Trans. Signal Process, 57(1), 92-106, (2009). DOI: 10.1109/TSP.2008.2005866.

29. Q. Wu, Y. D. Zhang, M. G. Amin and B. Himed. Complex multitask Bayesian compressive sensing. 2014 IEEE International Conference on Acoustics, Speech and Signal Processing (ICASSP), Florence, Italy, 3375-3379, (2014).

30. G. C. Cawley and N. L. C. Talbot. Sparse Bayesian learning and the relevance multi-layer perceptron network. Proceedings. 2005 IEEE International Joint Conference on Neural Networks, 2005., Montreal, QC, Canada, 2,1320-1324, (2005). DOI: 10.1109/IJCNN.2005.1556045.

31. S. Ji, Y. Xue and L. Carin. Bayesian Compressive Sensing. IEEE Transactions on Signal Processing, 56(6), 2346-2356, (2008). DOI: 10.1109/TSP.2007.914345.

32. MacKay and J. C. Da Vid. Bayesian interpolation. Neural Computation, 4(3), 415-447, (1992). DOI: 10.1162/neco.1992.4.3.415.

33. J. Li, X. Zhang and C. Han. Improved two-dimensional DOA estimation algorithm for two-parallel uniform linear arrays using propagator method. Signal Processing, 92(12), 3032-3038, (2012). DOI: 10.1016/j.sigpro.2012.06.010

34. Z. Yi, X. Xu, Y. A. Sheikh, et al. A rank-reduction based 2-D DOA estimation algorithm for three parallel uniform linear arrays. Signal Processing, 120(MAR.),305-310, (2016).

35. J. Li, D. Jiang and X. Zhang. Sparse representation based two-dimensional direction of arrival estimation using co-prime array. Multidimensional Systems and Signal Processing. 29, 35-47, (2018). 
Figures
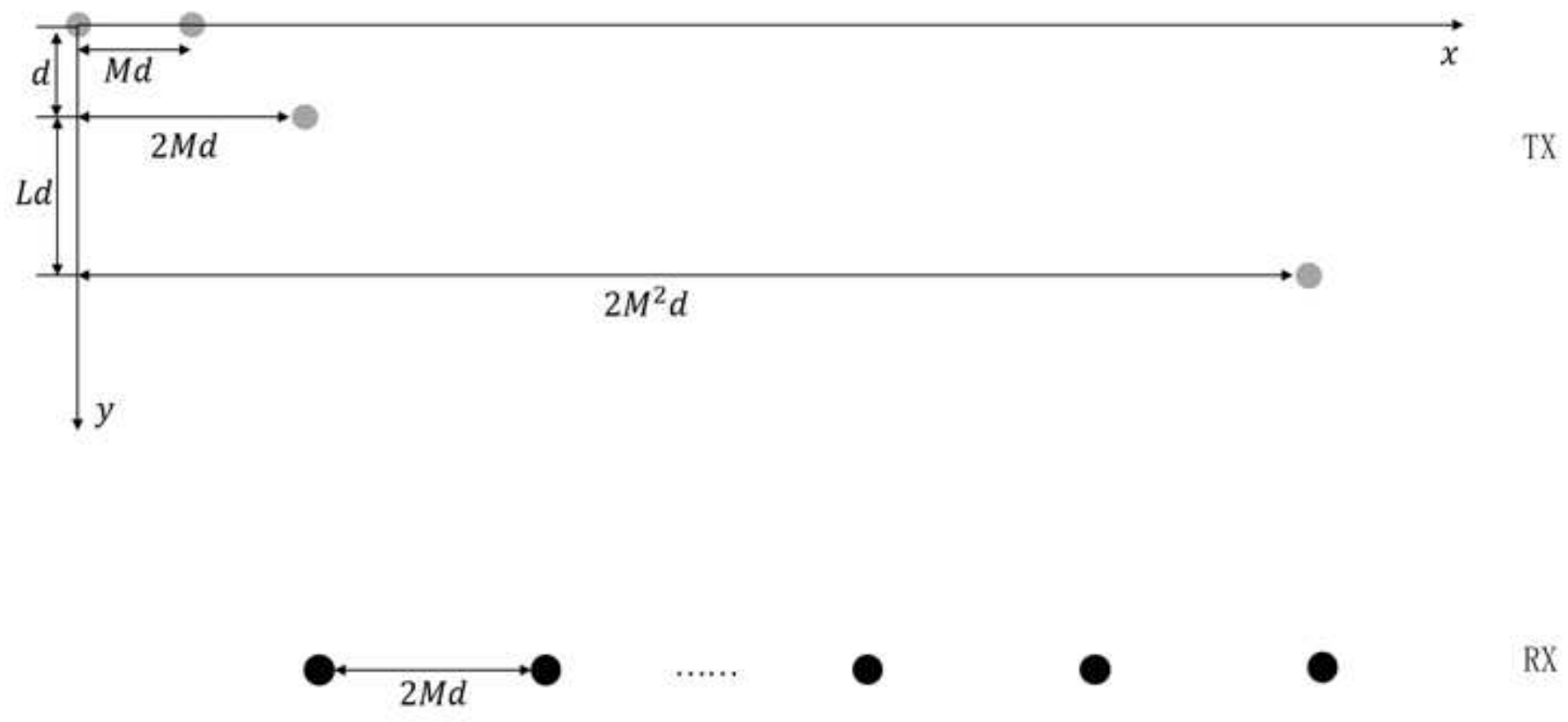

Figure 1

MIMO coprime array model

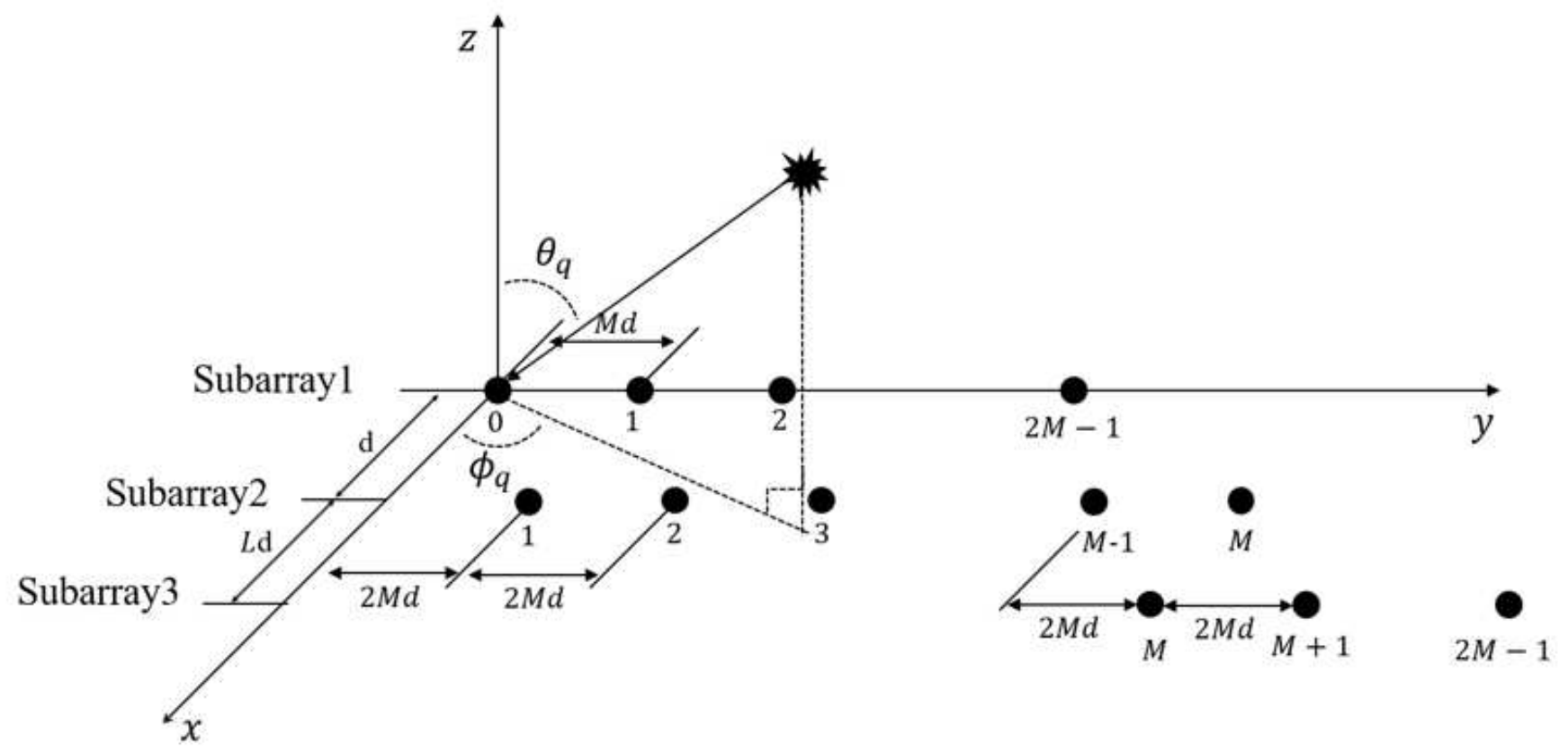

Figure 2

MIMO array model after virtual 


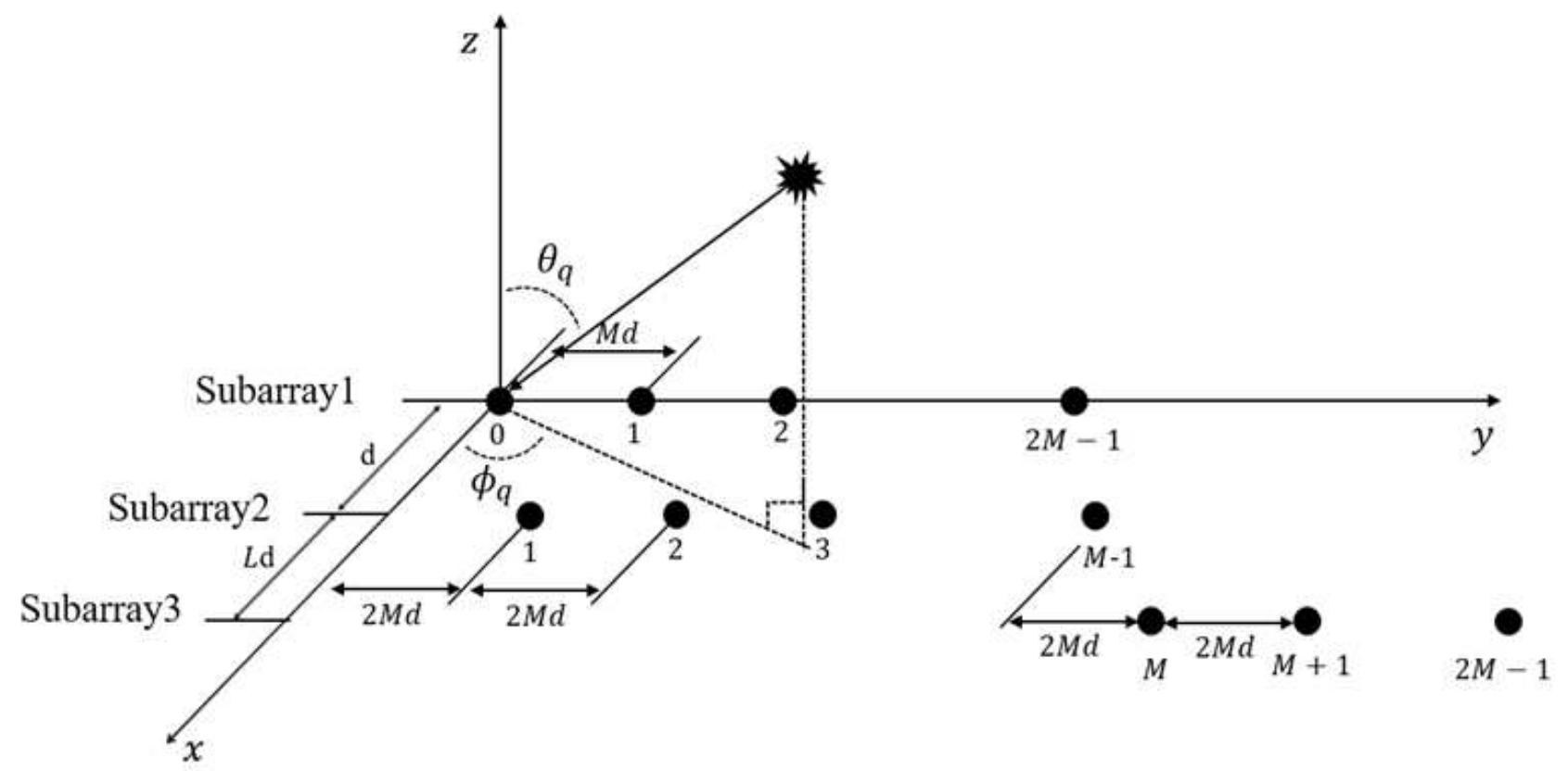

\section{Figure 3}

Virtual post-coprime MIMO array

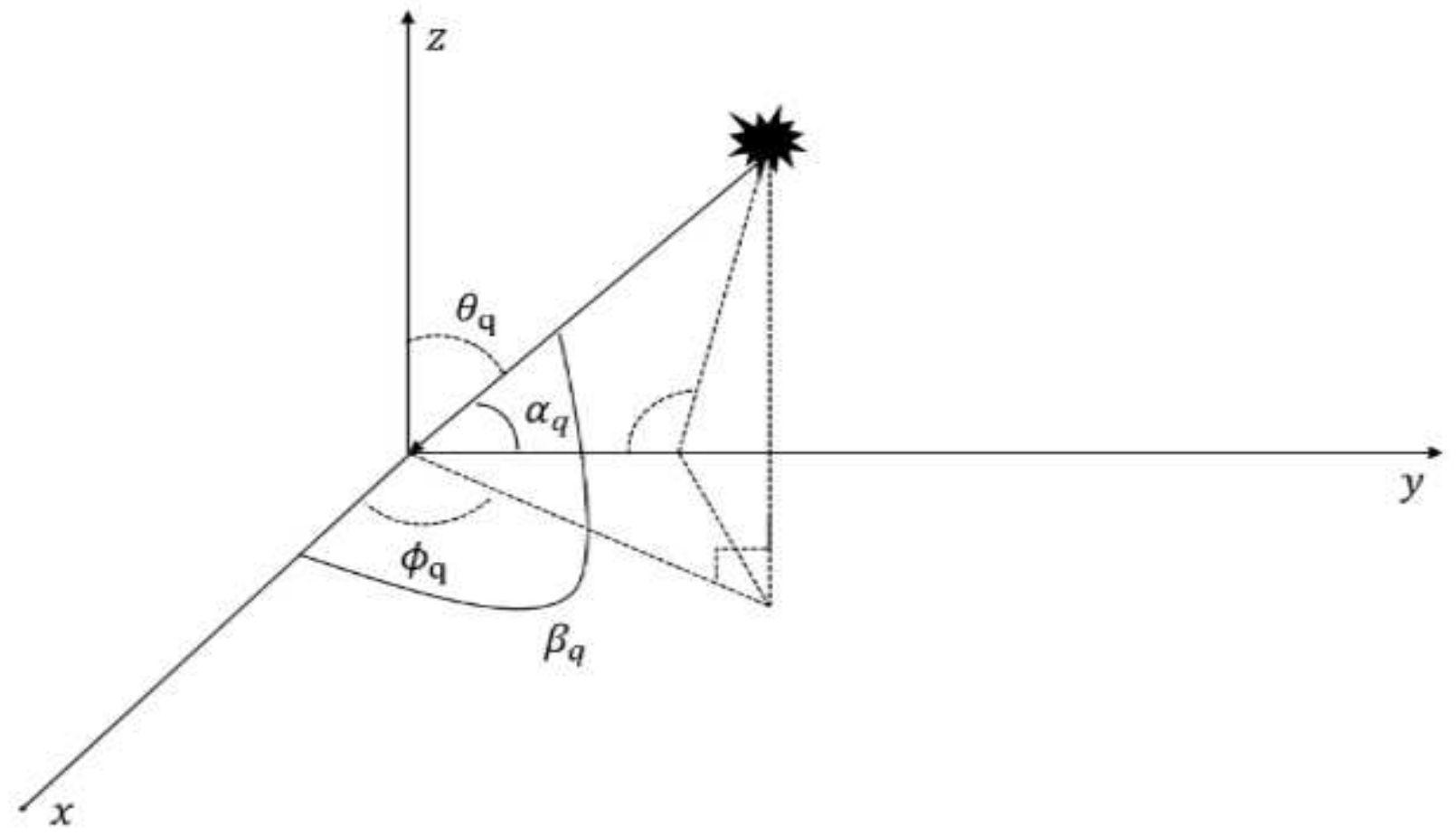

\section{Figure 4}

Please see the Manuscript PDF file for the complete figure caption 


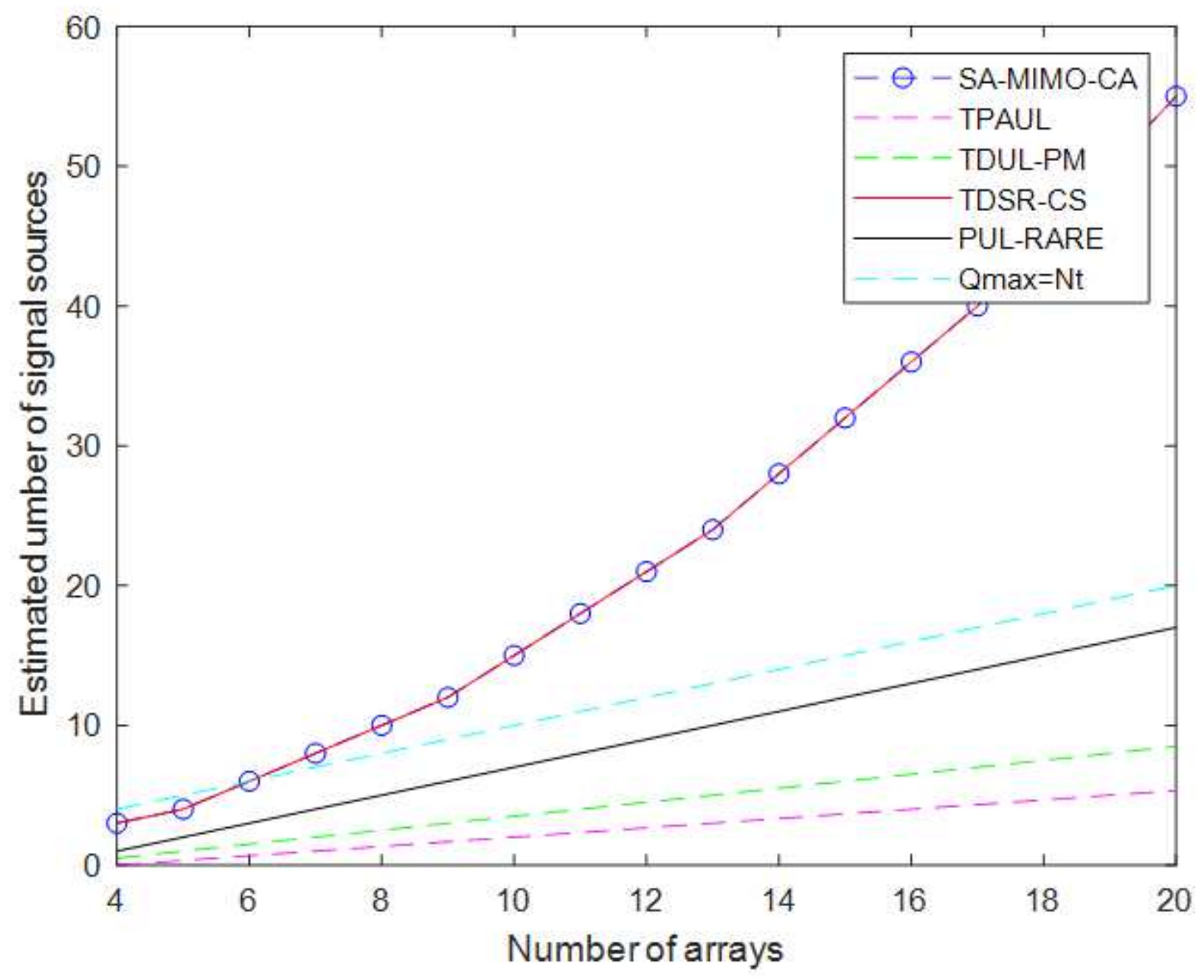

Figure 5

Please see the Manuscript PDF file for the complete figure caption

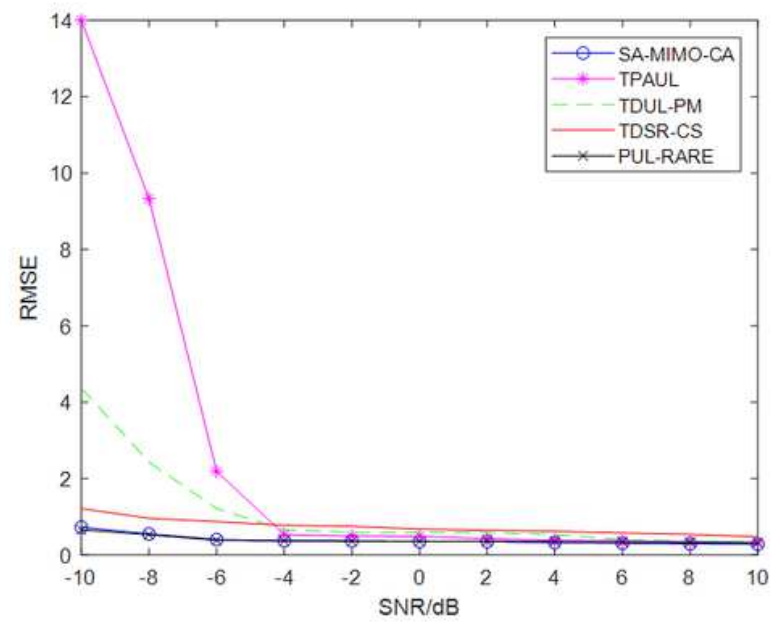

(a) The relationship between the SNR of the pitchlangle and the RMSE of the pitch angle

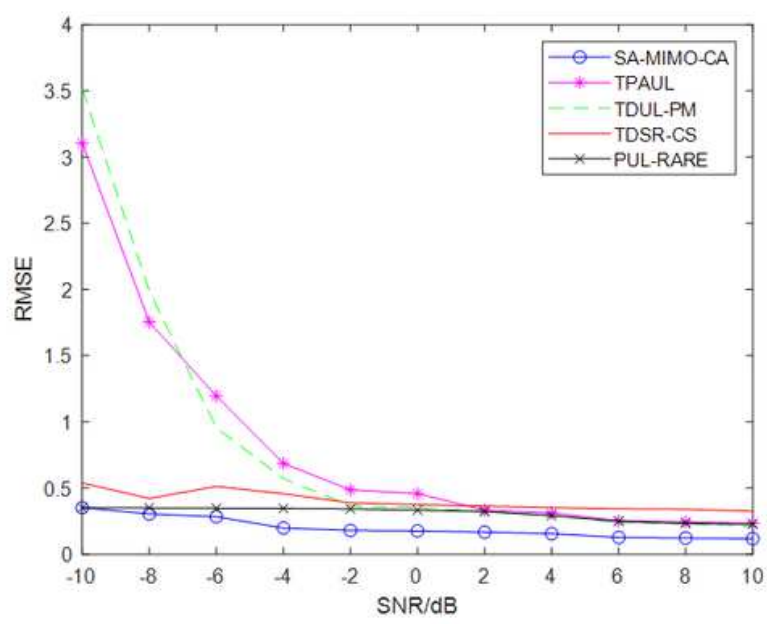

(b) The relationship between the SNR of the azimuth angle and RMSE of the azimuth angle 
Figure 6

The relationship between mean square error and signal-to-noise ratio (the number of sources is 3 )

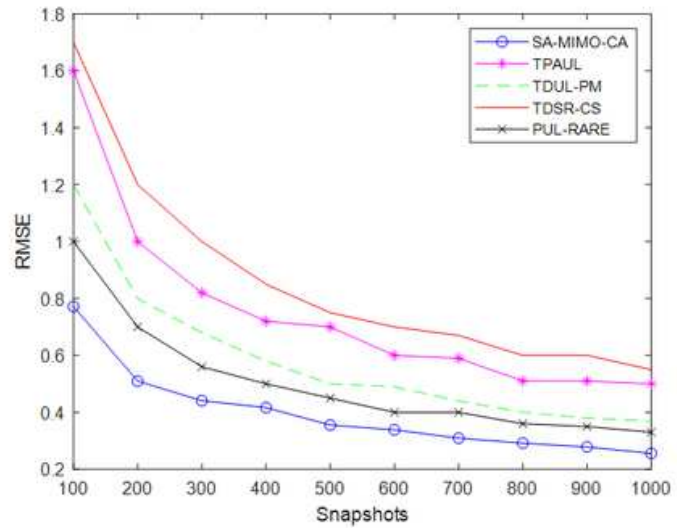

(a) The relationship between the mean square error of the pitch angle and the number of snapshots

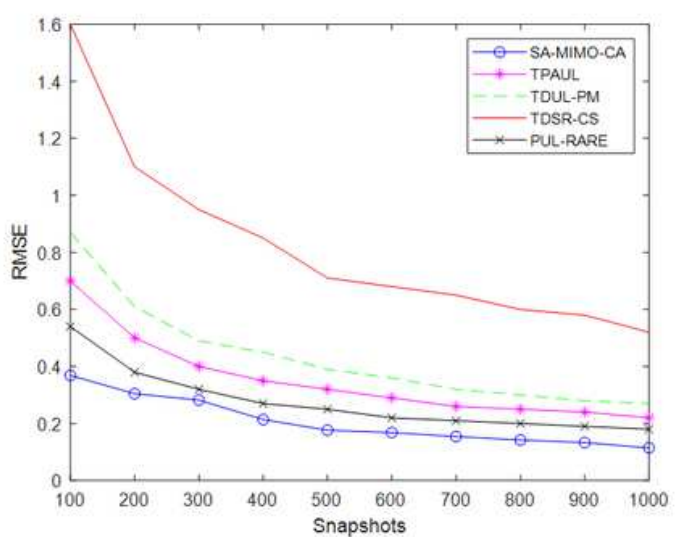

(b)The relationship between the mean square error of the azimuth angle and the number of snapshots

\section{Figure 7}

The relationship between the mean square error and the number of snapshots (the number of sources is 3)

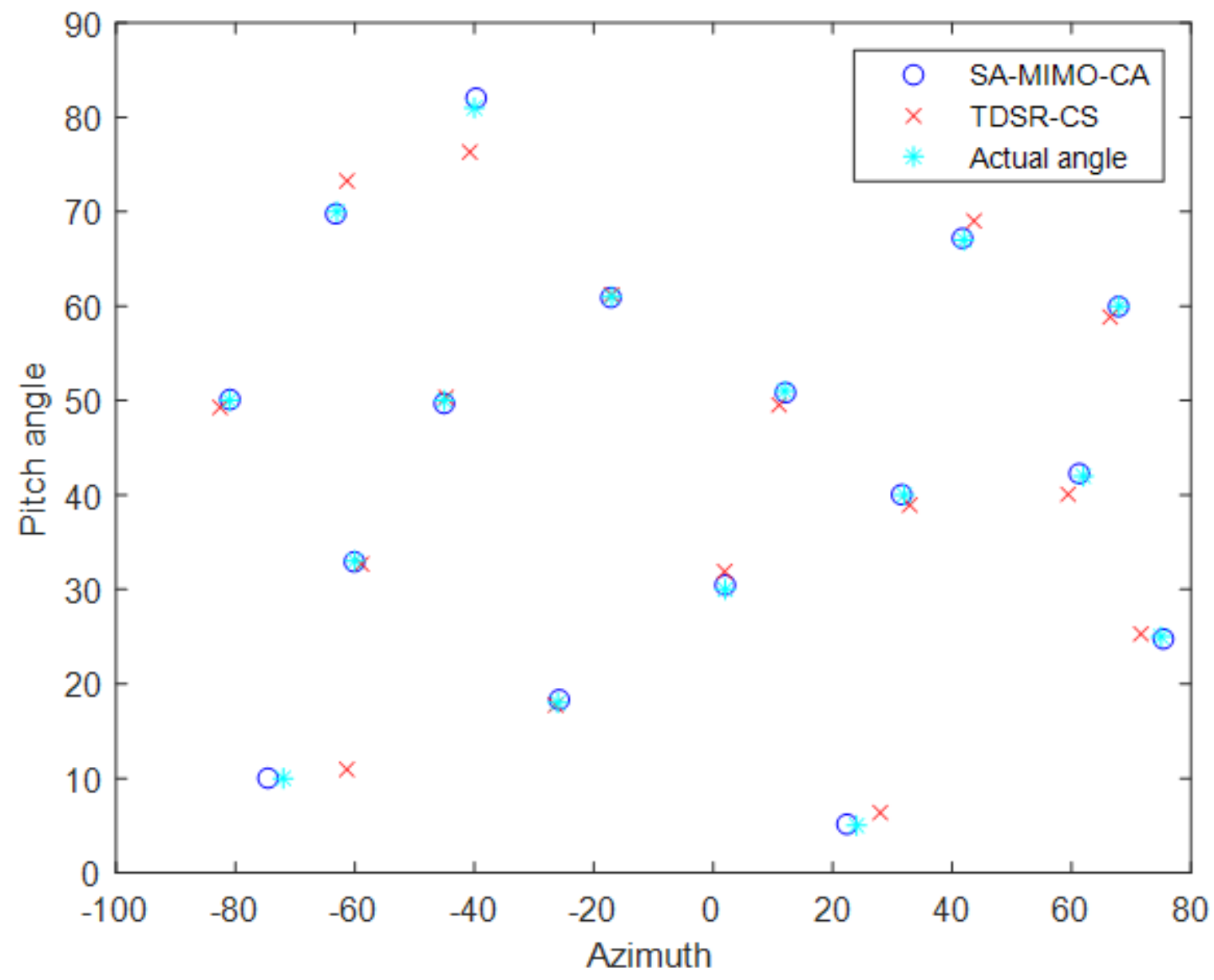


Figure 8

Comparison of two-dimensional DOA estimation 